\title{
STATIONARY STATES OF QUADRATIC DIFFUSION EQUATIONS WITH LONG-RANGE ATTRACTION*
}

\author{
MARTIN BURGER ${ }^{\dagger}$, MARCO DI FRANCESCO $^{\ddagger}$, AND MARZENA FRANEK ${ }^{\S}$
}

\begin{abstract}
We study the existence and uniqueness of nontrivial stationary solutions to a nonlocal aggregation equation with quadratic diffusion arising in many contexts in population dynamics. The equation is the Wasserstein gradient flow generated by the energy $E$, which is the sum of a quadratic free energy and the interaction energy. The interaction kernel is taken radial and attractive, nonnegative, and integrable, with further technical smoothness assumptions. The existence vs. nonexistence of such solutions is ruled by a threshold phenomenon, namely nontrivial steady states exist if and only if the diffusivity constant is strictly smaller than the total mass of the interaction kernel. In the one dimensional case we prove that steady states are unique up to translations and mass constraint. The strategy is based on a strong version of the Krein-Rutman Theorem. The steady states are symmetric with respect to their center of mass $x_{0}$, compactly supported on sets of the form $\left[x_{0}-L, x_{0}+L\right], C^{2}$ on their support, and strictly decreasing on $\left(x_{0}, x_{0}+L\right)$. Moreover, they are global minimizers of the energy functional $E$. The results are complemented by numerical simulations.
\end{abstract}

Key words. Nonlinear diffusion, nonlocal aggregation, population dynamics, stationary states, Krein-Rutman theorem.

AMS subject classifications. 35K65, 35B33, 35B41, 45K05, 45C05, 92D25, 92D50.

\section{Introduction}

Phenomena with long-range aggregation and short-range repulsion arise in many instances in population biology such as chemotaxis of cells, swarming or flocking of animals. A variety of mathematical models has been proposed for such situations, at the particle as well as at the continuum (mean field) level. In particular, if nonlocal repulsion acts at a smaller scale with respect to nonlocal attractive forces in the large particle limit, then a nonlocal repulsion term can be replaced by a local term with nonlinear diffusion; we refer to $[52,12,61,62,24$, $27,39,59,51,11,45]$ for several examples. A prototype model, which we shall also investigate further in this paper, is given by

$$
\partial_{t} \rho=\operatorname{div}(\rho \nabla(\varepsilon \rho-G * \rho)),
$$

where the convolution is carried out with an aggregation kernel $G$ such that $G(x)=g(|x|)$, with $g^{\prime}(r)>0$ for $r>0$. This model arises in a natural way as the limit of a stochastic interacting particle model with pair interactions (cf. $[53,54]$ and $[34,63,33]$ for general background). Models with the same structure have been recently used to model opinion formation; cf. [58, 60]. See also [22] and the recent $[32,26]$ for applications in imaging. Here we shall restrict to the case in which (1.1) is posed on the whole space $\mathbb{R}^{d}$.

${ }^{*}$ Received: February 13, 2012; accepted (in revised form): July 29, 2012. Communicated by Peter Markowich.

${ }^{\dagger}$ Institute for Computational and Applied Mathematics, Westfälische Wilhelms Universität (WWU) Münster, Einsteinstraße 62, D 48149 Münster, Germany (martin.burger@wwu.de).

${ }^{\ddagger}$ Departament de Matemàtiques, Universitat Autònoma de Barcelona, E-08193 - Bellaterra, Catalunya, Spain (mdifrance@gmail.com).

$\S$ Institute for Computational and Applied Mathematics, Westfälische Wilhelms Universität (WWU) Münster, Einsteinstraße 62, D 48149 Münster, Germany (marzena.franek@unimuenster.de). 
In case $G$ is $\lambda$-convex, then the equation (1.1) can be formulated as a gradient flow in the Wasserstein metric (cf. $[1,65,66]$ ) of the energy (or entropy) functional

$$
E[\rho]:=\frac{\varepsilon}{2} \int_{\mathbb{R}^{d}} \rho^{2}(x) d x-\frac{1}{2} \int_{\mathbb{R}^{d}} \int_{\mathbb{R}^{d}} G(x-y) \rho(y) \rho(x) d y d x ;
$$

see $[50,4,19,20]$. Several properties of models of the above form have been considered in the literature, e.g. existence and uniqueness in the context of entropy solutions [13], $H^{-1}$ solutions [9], well-posedness in the Wasserstein gradient flow sense within the theory developed in [1], and - in particular in connection with the classical Patlak-Keller-Segel model for chemotaxis [57, 40]-with respect to blow-up vs. large-time existence; cf. e.g. [38, 35, 10, 25] for models with linear diffusion and $[42,16,41]$ for models with nonlinear diffusion.

An interesting and important question is the characterization of large-time behavior of solutions to equations of the form (1.1), which is related with the possible existence of nontrivial steady states, even when the quadratic diffusion is replaced by a more general nonlinear diffusion. This issue is solved in detail for purely diffusive equations (no aggregation term), in which solutions decay to zero with a prescribed rate for large times and behave like the (compactly supported) Barenblatt profiles; cf. the classical works of Vazquez on the self-similar behavior of the porous medium equation, which are nicely collected in the book [64], as well as the papers $[55,21]$. In the purely nonlocal case, namely when $\varepsilon=0$, this issue has been studied extensively in many papers $[46,14,44,6,5,17,18,7,8$, $37,30,29,2]$, combined with the study of the regularity of solutions compared to the attractive singularity of the interaction kernel. In particular, solutions are known to concentrate to a Dirac delta centered at the initial center of mass (invariant) either in a finite or in an infinite time, depending on the properties of the kernel $G$ at $x=0$. When the kernel $G$ is supported on the whole space, the Dirac delta is the unique steady state of (1.1) with unit mass and zero center of mass.

The asymptotic behavior in the general case with both nonlinear diffusion and nonlocal interaction has been only partially addressed. A first attempt in this direction was performed in [14], in which the existence of steady states of (1.1) for sufficiently small $\varepsilon$ and the non-existence for large $\varepsilon$ in the one-dimensional case was proven by means of the pseudo-inverse representation of the Wasserstein distance. More refined results in a similar model derived in $[36,56]$ with cutoff density have been found in $[15,43]$. Parallel to their work, the authors of the present paper recovered the results in [3], in which a quasi sharp result of existence of minimizers for the energy $E[\rho]$ in a multi dimensional framework has been proven.

A key open (to our knowledge) problem in this context is the uniqueness of steady states under mass and center of mass constraint, its main difficulty being the fact that the functional $E$ is neither convex in the classical sense nor in the displacement convex sense [50] (except when $G$ is globally concave on $\mathbb{R}^{d}$; see [19]) when $\varepsilon<\int G$ with $G \geq 0$.

In this paper we further investigate the detailed structure of steady states in one space dimension. We remark that our work does not go into the direction of providing sharp regularity conditions on the kernel $G$. Roughly speaking, $G$ is smooth, radial (with decreasing profile), nonnegative, integrable, and supported on the whole space. The precise assumptions on $G$ are stated at the beginning 
of Section 2. We found out that for a kernel $G$ decreasing on $x>0$, the $L^{1}$-norm of $G$ compared to $\varepsilon$ marks a threshold:

- If $\varepsilon \geq \int G$, then there exists no steady state.

- If $\varepsilon<\int G$, there exists a stationary state.

The same results are recovered in [3], except for the critical case $\varepsilon=\int G$.

The main result of our paper deals with the case $d=1$. Here we can give a detailed characterization of the stationary states in the case $\varepsilon<\int G$. If $G^{\prime}$ only vanishes at zero, then there exists a unique stationary state (up to translation and mass normalization), which is a minimizer of the energy $E$ at fixed mass. The stationary states have compact support, which increases with $\varepsilon$. In particular, the size of the support can be made arbitrarily large as $\varepsilon$ grows to $\int G$, and arbitrarily small as $\varepsilon$ decreases to zero. Moreover, such a steady state is symmetric with a single maximum at its center of mass $x_{c}$, and monotonically decreasing on $x>x_{c}$. The precise statement can be found in Theorem 4.13. If $\varepsilon$ is small enough, the stationary states are concave on their support. The most important tool in the proof of the main result is the statement of the stationary equation as an eigenvalue problem (see formula (4.10)), together with the use of a strong version of the Krein-Rutman Theorem (cf. Theorem 4.10), which allows one to characterize the steady states as eigenfunctions corresponding to the principal eigenvalue of a strongly positive compact operator defined on $C^{2}$ functions on a given interval $[-L, L]$; see Proposition 4.11. A key property in this sense (namely the monotonicity of the principal eigenvalue $\varepsilon=\varepsilon(L)$ ) is proven in Proposition 4.12. After a first version of the present paper, [31] studied a very special repulsive-attractive equation without diffusion and proved a uniqueness result using a similar method. In this respect, we stress the fact that our result holds for a wide class of kernels $G$ (in particular, we do not assume any homogeneity of $G$ ). More recently, the authors have realized that Chayes et al. [23] have investigated the large time behaviour of (1.1) on the torus in the diffusion dominated case, i.e. in cases in which the constant steady state is stable.

The uniqueness of the stationary state in this context is somewhat surprising, since in our case the energy functional $E$ is not convex for $\varepsilon<\int G$, and thus one might expect other stationary points of $E$. On the other hand also in the case $\varepsilon=0$ one can see that there is a unique (measure) stationary state concentrated at the center of mass if $G$ has global support. Adding the squared norm for positive $\varepsilon$ makes the functional closer to convex, and thus probably does not lead to additional stationary points. Similar to the case $\varepsilon=0$, we expect that multiple steady states arise if $G$ is compactly supported; see Remark 4.15 .

The paper is organized as follows. In Section 2 we recall the statement of the problem and provide some preliminary regularity results. In Section 3 we complement our results with those proven in [3] and provide sharp conditions on $\varepsilon$ and $\int G$ for the existence of non trivial steady states. In Section 4 we prove our main results about the uniqueness of steady states in one space dimension. Finally, in Section 5 we complement our results with some numerical simulations.

\section{Preliminaries}

We consider the evolution equation

$$
\partial_{t} \rho=\operatorname{div}(\rho \nabla(\varepsilon \rho-G * \rho))
$$


and its stationary version

$$
0=\operatorname{div}(\rho \nabla(\varepsilon \rho-G * \rho)),
$$

posed on the whole space $\mathbb{R}^{d}$. Due to the applied setting of the model, we shall consider here only nonnegative solutions $\rho \geq 0$.

Assumptions on the kernel $G$. We shall assume throughout the paper that the interaction kernel $G$ satisfies

1. $G \geq 0$, and $\operatorname{supp}(G)=\mathbb{R}^{d}$;

2. $G \in W^{1,1}\left(\mathbb{R}^{d}\right) \cap L^{\infty}\left(\mathbb{R}^{d}\right) \cap C^{2}\left(\mathbb{R}^{d}\right)$;

3. $G(x)=g(|x|)$ for all $x \in \mathbb{R}^{d}$;

4. $g^{\prime}(r)<0$ for all $r>0$;

5. $g^{\prime \prime}(0)<0$,

6. $\lim _{r \rightarrow+\infty} g(r)=0$.

We emphasize here that providing sharp conditions on the regularity of $G$ is not a purpose of the present paper. Let us recall that the equation (2.1) preserves the total mass

$$
M=\int \rho(x) d x
$$

and center of mass

$$
C M[\rho(t)]:=\int x \rho(x, t) d x .
$$

Moreover, it is easily seen that, for a given stationary state $\rho$ solving $(2.2), M \rho$ and $\rho\left(\cdot+x_{0}\right)$ are still stationary states for all $M>0$ and $x_{0} \in \mathbb{R}$.

We shall therefore assume $M=1$ for simplicity. For future use we introduce the space

$$
\mathcal{P}=\left\{\rho \in L_{+}^{1}\left(\mathbb{R}^{d}\right): \int_{\mathbb{R}^{d}} \rho(x) d x=1\right\} .
$$

Moreover, from now on we shall assume for simplicity

$$
\|G\|_{L^{1}}=\int G(x) d x=1 .
$$

This is not restrictive since the kernel $G$ can always be normalized by modifying the diffusion constant and the time scale as follows

$$
\begin{aligned}
& \partial_{\tau} \rho=\operatorname{div}\left(\rho \nabla\left(\varepsilon^{\prime} \rho-\widetilde{G} * \rho\right)\right), \\
& \tau=\|G\|_{L^{1}\left(\mathbb{R}^{d}\right)}, \quad \widetilde{G}=G /\|G\|_{L^{1}\left(\mathbb{R}^{d}\right)}, \quad \varepsilon^{\prime}=\varepsilon /\|G\|_{L^{1}\left(\mathbb{R}^{d}\right)} .
\end{aligned}
$$

Let us recall the following results on the existence and uniqueness of gradient flow solutions to (2.1), which follows from the theory developed in [1]. In this sense, let us introduce one of the most important tools related to the study of the evolution equation (2.1), and in particular with the stationary version (2.2), namely the energy functional

$$
E[\rho]:=\frac{\varepsilon}{2} \int_{\mathbb{R}^{d}} \rho^{2}(x) d x-\frac{1}{2} \int_{\mathbb{R}^{d}} \int_{\mathbb{R}^{d}} G(x-y) \rho(y) \rho(x) d y d x .
$$


Theorem 2.1 ([1]). Let $\rho_{0} \in L^{2} \cap \mathcal{P}$ such that $\rho \geq 0$ and $E\left[\rho_{0}\right]<+\infty$. Let $G$ satisfy the above assumptions. Then there exists a unique weak solution $\rho$ to (2.1) with

- $E[\rho(t)]<+\infty$ for all $t \geq 0$;

- $\sqrt{\rho} \nabla(\varepsilon \rho-G * \rho) \in L^{2}\left([0, T] \times \mathbb{R}^{2}\right)$ for all $T>0$,

such that the following energy identity is satisfied:

$$
E[\rho(t)]+\int_{0}^{T} \int_{\mathbb{R}^{d}} \rho|\nabla(\varepsilon \rho-G * \rho)|^{2} d x d t=E\left[\rho_{0}\right] .
$$

In particular, the equation (2.1) produces the following regularizing effect.

Lemma 2.2 (Regularity of $L^{2} \cap \mathcal{P}$ steady states). Let $\rho_{0} \in L^{2} \cap \mathcal{P}$. Then the corresponding solution $\rho(t)$ to $(2.1)$ satisfies

$$
\int \rho|\nabla \rho|^{2} d x<+\infty
$$

for almost every $t>0$. In particular, if $\rho$ be an $L^{2} \cap \mathcal{P}$ steady state to (2.1), then $\rho$ satisfies (2.4) and $\rho \in C^{2}$ on $\operatorname{supp}[\rho]$.

Proof. Due to (2.3), the quantity

$$
\varepsilon \int \rho|\nabla \rho|^{2} d x-2 \varepsilon \int \rho \nabla \rho \cdot \nabla G * \rho d x+\int \rho|\nabla G * \rho|^{2} d x
$$

is finite for almost every $t>0$, and therefore, in view of Cauchy-Schwarz inequality, we have

$$
\frac{\varepsilon}{2} \int \rho|\nabla \rho|^{2} d x-C(\varepsilon) \int \rho|\nabla G * \rho|^{2} d x+\int \rho|\nabla G * \rho|^{2} d x<+\infty,
$$

and thanks to the smoothness assumptions on $G$ we have the assertion (2.4). If $\rho$ be a steady state, then $\rho$ satisfies (2.4) too. This implies in particular that $\nabla \rho$ is almost everywhere finite on $\mathbb{R}^{2}$. The energy identity (2.3) implies then

$$
\rho|\nabla(\varepsilon \rho-G * \rho)|^{2}=0
$$

for almost every $x \in \mathbb{R}^{2}$. This means that

$$
\varepsilon \rho-G * \rho=\text { constant }
$$

almost everywhere on every connected component of the support of $\rho$. By convolution with standard mollifiers, one can easily see that $\varepsilon \rho-G * \rho=C$ for a given $C$ depending on the connected component of $\operatorname{supp}[\rho]$. Since $G$ is $C^{2}$, this easily implies $\rho \in C^{2}$ on $\operatorname{supp}[\rho]$.

Corollary 2.3 (1d regularity). Let $\rho$ be an $L^{2} \cap \mathcal{P}$ solution to (2.2) in one space dimension. Then $\rho$ is continuous on $\mathbb{R}$.

Proof. Apply the result in Lemma 2.2 to the case $d=1$. Since $\frac{d}{d x} \rho^{3 / 2} \in L^{2}$, the one dimensional Sobolev embedding implies that $\rho^{3 / 2}$ is continuous. 


\section{Stationary solutions in multiple dimensions}

In this section we state the necessary and sufficient conditions on $\varepsilon$ and $\|G\|_{L^{1}}$ such that there exist non trivial steady states

$$
\rho \nabla(\varepsilon \rho-G * \rho)=0
$$

in the set $L^{2} \cap \mathcal{P}$. During our work, we realized that J. Bedrossian has obtained similar results in [3], based on ideas and strategies developed in [47] and [49]. In order to simplify the coverage of the paper, we shall state the result in $[3$, Theorem 1] and prove all other results. Notice that the critical case $\varepsilon=\|G\|_{L^{1}}$ was not covered in [3].

Let us start by focusing on the interplay between the solutions to (3.1) and the variational calculus on the energy functional

$$
E[\rho]:=\varepsilon \int_{\mathbb{R}^{d}} \rho^{2}(x) d x-\frac{1}{2} \int_{\mathbb{R}^{d}} \int_{\mathbb{R}^{d}} G(x-y) \rho(y) \rho(x) d y d x .
$$

In the next proposition we prove that being a minimum for the energy functional is a sufficient condition for being a solution to (3.1).

Proposition 3.1 (Stationary solutions via energy minimization). Let $\rho \in$ $L^{2}\left(\mathbb{R}^{d}\right)$ be a minimizer for the energy functional

$$
E[\rho]:=\frac{1}{2} \int_{\mathbb{R}^{d}} \rho(\varepsilon \rho-G * \rho) d x
$$

on $\mathcal{P}$. Then

$$
\rho \nabla(\varepsilon \rho-G * \rho)=0 \quad \text { a. e. in } \mathbb{R}^{d} .
$$

Proof. Let $V \in C_{c}^{1}\left(\mathbb{R}^{d}\right)$ be an arbitrary vector field and let $u(x, s)$ be a local solution to the continuity equation

$$
\partial_{s} u(x, s)+\operatorname{div}(u(x, s) V(x))=0
$$

with initial datum

$$
u(x, 0)=\rho(x),
$$

with $\rho$ being the minimizer for $E$ given in the hypothesis. Such a $u$ can be constructed by solving the characteristic ODE

$$
\frac{d}{d s} X(x, s)=V(X(x, s))
$$

coupled with the initial datum

$$
X(x, 0)=x
$$

locally in $s=0$, with the local solution $X(x, s)$ being $C^{1}$, and by taking $u(x, s):=$ $\left[(X(\cdot, s))_{\sharp} \rho\right](x, s)$, i.e. $u(x, s)$ defined via

$$
\int \phi(x) u(x, s) d x=\int \phi(X(x, s)) \rho(x) d x, \quad \text { for all } \phi \in C_{c}^{1}\left(\mathbb{R}^{d}\right)
$$


(cf. for instance [1, Chapter 8, Lemma 5.5.3]). For all $s$ in the interval of existence of $u$ we have

$$
\int u(x, s) d x=1, \quad u(x, s) \geq 0 \quad \text { a.e., }
$$

and therefore the map $s \mapsto E[u(\cdot, s)]$ has a local minimum at $s=0$. Hence

$$
\begin{aligned}
0 & \leq\left.\frac{d}{d s} E[u(\cdot, s)]\right|_{s=0}=\left.\int(\varepsilon u-G * u) \partial_{s} u d x\right|_{s=0} \\
& =-\left.\int(\varepsilon u-G * u) \operatorname{div}(u V) d x\right|_{s=0}=\int \rho \nabla(\varepsilon \rho-G * \rho) \cdot V d x,
\end{aligned}
$$

and by replacing $V$ with $-V$ we obtain

$$
0 \geq \int \rho \nabla(\varepsilon \rho-G * \rho) \cdot V d x
$$

and therefore

$$
\int \rho \nabla(\varepsilon \rho-G * \rho) \cdot V d x=0, \quad \text { for an arbitrary } V \in C_{c}^{1}\left(\mathbb{R}^{d}\right),
$$

which is the desired assertion.

Let us now compute the first and the second order Gâteaux derivatives of $E$.

Lemma 3.2. Let $\rho \in L^{2} \cap \mathcal{P}$ be a solution to (3.1). Then, $\rho$ is a stationary point for the energy functional $E$. Moreover, the second order Gâteaux derivative of $E$ on $\rho$ satisfies

$$
\left.\frac{d^{2}}{d \delta^{2}} E[\rho+\delta v]\right|_{\delta=0}=\varepsilon \int_{\mathbb{R}^{d}} v^{2}(x) d x-\int v(x) G * v(x) d x,
$$

for all $v=\operatorname{div}(\rho V)$ and $V \in C_{c}^{1}\left(\mathbb{R}^{d}\right)$.

Proof. Suppose $\rho \in L^{2} \cap \mathcal{P}$ satisfies (3.1). Let us compute

$$
\lim _{\delta \rightarrow 0} \frac{1}{\delta}(E[\rho+\delta v]-E[\rho]),
$$

with $v=\operatorname{div}(\rho V)$ for an arbitrary vector field $V \in C_{c}^{1}$ (so that $\int v(x) d x=0$ ). We obtain

$$
\begin{aligned}
& \frac{1}{\delta}(E[\rho+\delta v]-E[\rho]) \\
= & \frac{\varepsilon}{2 \delta} \int_{\operatorname{supp}(\rho+\delta v)}(\rho+\delta v)^{2} d x-\frac{\varepsilon}{2 \delta} \int_{\operatorname{supp}(\rho)} \rho^{2} d x \\
& -\frac{1}{2 \delta} \int_{\operatorname{supp}(\rho+\delta v)}(\rho+\delta v) G *(\rho+\delta v) d x+\frac{1}{2 \delta} \int_{\operatorname{supp}(\rho)} \rho G * \rho d x .
\end{aligned}
$$

Therefore we easily get

$$
\begin{aligned}
& \lim _{\delta \rightarrow 0} \frac{1}{\delta}(E[\rho+\delta v]-E[\rho])=\int v(\varepsilon \rho-G * \rho) d x \\
= & \int \operatorname{div}(\rho V)(\varepsilon \rho-G * \rho) d x=-\int \rho V \cdot \nabla(\varepsilon \rho-G * \rho) d x .
\end{aligned}
$$


Therefore, $\rho$ is a stationary point for $E$ under the constraint $\int \rho d x=1$. The computation of the second derivative of $E$ on $\rho$ yields

$$
\begin{aligned}
\frac{d^{2}}{d \delta^{2}} E[\rho+\delta v]= & \frac{d^{2}}{d \delta^{2}} \frac{\varepsilon}{2} \int_{\operatorname{supp}(\rho+\delta v)}(\rho+\delta v)^{2} d x \\
& \quad-\frac{d^{2}}{d \delta^{2}} \frac{1}{2} \int_{\operatorname{supp}(\rho+\delta v)}(\rho+\delta v) G *(\rho+\delta v) d x \\
= & \varepsilon \int v^{2} d x-\int v G * v d x,
\end{aligned}
$$

which is independent of $\delta$ and therefore it is valid also for $\delta=0$.

Before we start analyzing the existence or nonexistence of steady states, we introduce a very simple technical lemma which will be very useful in the sequel.

Lemma 3.3. Suppose $\rho \in L^{2} \cap \mathcal{P}$ is a solution to (3.1) having connected support. Then

$$
\varepsilon \rho(x)=\int_{\operatorname{supp}[\rho]} G(x-y) \rho(y) d y+C
$$

for all $x \in \operatorname{supp}[\rho]$ with $C=2 E[\rho]$. Moreover, if $\operatorname{supp}[\rho]$ has infinite measure, then $C=E[\rho]=0$.

Proof. It is immediate from (3.1) that

$$
\varepsilon \rho(x)=\int_{\operatorname{supp}[\rho]} G(x-y) \rho(y) d y+C
$$

for all $x \in \operatorname{supp}[\rho]$ and a certain constant $C$. Then, we multiply (3.3) by $\rho(x)$ and integrate over $\operatorname{supp}[\rho]$ to obtain

$$
\varepsilon \int_{\operatorname{supp}[\rho]} \rho^{2}(x) d x=\int_{\operatorname{supp}[\rho]} \int_{\operatorname{supp}[\rho]} G(x-y) \rho(y) \rho(x) d y d x+C,
$$

where we have used that $\rho$ has unit mass. It is therefore clear that $C=2 E[\rho]$. Suppose now that $\operatorname{supp}[\rho]$ has infinite measure. Suppose by contradiction that $C \neq 0$. Let $\left\{x_{k}\right\} \subset \operatorname{supp}[\rho]$ be a sequence of points such that $\left|x_{k}\right| \rightarrow+\infty$. We have, for all $k$,

$$
\varepsilon \rho\left(x_{k}\right)-\int_{\operatorname{supp}[\rho]} G\left(x_{k}-y\right) \rho(y) d y=C,
$$

and therefore the same expression should hold in the limit $k \rightarrow+\infty$. Now, the assumptions on $G$ imply that the integral

$$
\int_{\operatorname{supp}[\rho]} G\left(x_{k}-y\right) \rho(y) d y
$$

converges to zero as $k \rightarrow+\infty$. This is due to Lebesgue's dominated convergence Theorem. Therefore, the term $\rho\left(x_{k}\right)$ has a limit $C$ as $k \rightarrow+\infty$. Such limit is the same for all diverging sequences of points $\left\{x_{k}\right\} \subset \operatorname{supp}[\rho]$, which means

$$
\lim _{x \in \operatorname{supp}[\rho],|x| \rightarrow+\infty} \rho(x)=C .
$$

Now, since $\operatorname{supp}[\rho]$ has infinite measure, then $C \neq 0$ implies that $\rho$ is not integrable, which is a contradiction. Therefore $C=0$. 
3.1. Non-existence of nontrivial steady states for $\varepsilon>1$. We start by covering the case $\varepsilon>1$. Here, there exist no nontrivial steady states, as it follows from the following simple lemma.

Lemma 3.4. Let $\varepsilon>1$. Then, there exist no stationary solutions to (3.1) in the space $L^{2} \cap \mathcal{P}$.

Proof. We first prove that there exists no minimizer for $E[\rho]$ under the mass constraint $\int \rho=1$ and $\rho \geq 0$. To see this, we use Young's inequality for convolutions as follows:

$$
E[\rho]=\frac{\varepsilon}{2} \int \rho^{2} d x-\frac{1}{2} \int \rho G * \rho d x \geq \frac{\varepsilon}{2} \int \rho^{2} d x-\frac{\|G\|_{L^{1}}}{2} \int \rho^{2} d x=\frac{\varepsilon-1}{2} \int \rho^{2} d x
$$

with $\varepsilon-1>0$. Moreover, we have the simple estimate $E[\rho] \leq C\|\rho\|_{L^{2}}^{2}$. Take a family of functions $\rho_{\lambda}(x) \geq 0$ such that $\int \rho_{\lambda}(x) d x=1$ and $\int \rho_{\lambda}^{2}(x) d x \rightarrow 0$ as $\lambda \rightarrow$ $+\infty$. To construct such a family, we just take a fixed $L_{+}^{2}\left(\mathbb{R}^{d}\right)$ function $\rho \not \equiv 0$ and rescale it by $\rho_{\lambda}(x)=\lambda^{-d} \rho\left(\lambda^{-1} x\right)$. For such a family we therefore have

$$
E\left[\rho_{\lambda}\right] \rightarrow 0, \quad \text { as } \lambda \rightarrow \infty .
$$

Therefore, it is impossible to have a minimizer $\rho_{\infty}$ for $E[\rho]$ in the set $\left\{\rho \in L_{+}^{1}: \int \rho=1\right\}$ because (3.4) would imply that $E\left[\rho_{\infty}\right]>0$, and we would necessarily have $0<E\left[\rho_{\lambda}\right]<E\left[\rho_{\infty}\right]$ for $\lambda$ large enough.

Now we prove that there exist no steady states. Suppose by contradiction that $\rho$ is a steady state. Then, due to Lemma 3.2, $\rho$ is a stationary point for $E$. Moreover, the formula (3.2) implies that the functional $E$ is strongly convex, and therefore admits only one stationary point, which coincides with its global minimizer. But this contradicts the non-existence of a global minimizer proven above.

3.2. The critical case $\varepsilon=1$. We aim to solve

$$
0=\operatorname{div}(\rho \nabla(\rho-G * \rho)) .
$$

We shall prove that no $L^{2} \cap \mathcal{P}$ steady states exist in this case.

TheOREm 3.5 (Non-existence of nontrivial steady states for $\varepsilon=1$ ). There exist no solutions to $(3.5)$ in $L^{2} \cap \mathcal{P}$.

Proof. From the Cauchy-Schwartz inequality we know that

$$
\int_{\mathbb{R}^{d}} \rho G * \rho d x \leq\|\rho\|_{L^{2}\left(\mathbb{R}^{d}\right)}\|G * \rho\|_{L^{2}\left(\mathbb{R}^{d}\right)},
$$

and the equality in the above formula holds if and only if $\rho$ and $G * \rho$ are proportional. In terms of the functional $E$ this means that

$$
E[\rho] \geq 0 \quad \text { for all } \rho \in L^{2}\left(\mathbb{R}^{d}\right) \cap \mathcal{P}\left(\mathbb{R}^{d}\right) .
$$

As in Lemma 3.4, we have the estimate $E[\rho] \leq C\|\rho\|_{L^{2}}^{2}$, and using once again the family $\rho_{\lambda}$ of Lemma 3.4 we see that $\inf _{\rho \in L^{2} \cap \mathcal{P}} E[\rho]=0$. Assume by contradiction that there exists a stationary solution $\rho_{\infty}$. Then, due to the result in Lemma 3.2 and in view of the Cauchy-Schwartz inequality, the second order derivative of $E$ is nonnegative everywhere. Hence, the functional $E$ is convex and therefore 
$\rho_{\infty}$ is a global minimizer for $E$ under the constraint $\rho \in L^{2} \cap \mathcal{P}$. Then, we must have $E\left[\rho_{\infty}\right]=0$, which means that $\rho$ and $G * \rho$ are proportional, i.e. there exists a constant $\lambda \in \mathbb{R}_{+}$such that

$$
\rho_{\infty}(x)=\lambda G * \rho_{\infty}(x)
$$

almost everywhere on $\mathbb{R}^{d}$. Integrating (3.6) over $\mathbb{R}^{d}$ yields

$$
1=\lambda\|G\|_{L^{1}\left(\mathbb{R}^{d}\right)}=\lambda,
$$

and hence

$$
\rho_{\infty}(x)=G * \rho_{\infty}(x)
$$

almost everywhere on $\mathbb{R}^{d}$. We can then apply the Fourier transform

$$
\widehat{f}(\xi)=\int_{\mathbb{R}^{d}} e^{-2 \pi i x \cdot \xi} f(x) d x
$$

to both members of the equation (3.7) to obtain

$$
\widehat{\rho_{\infty}}(\xi)=\widehat{G}(\xi) \widehat{\rho_{\infty}}(\xi), \quad \xi \in \mathbb{R}^{d} .
$$

We have

$$
|\widehat{G}(\xi)| \leq \int_{\mathbb{R}^{d}}|G(x)| d x=1 .
$$

Moreover, since $G$ is even, then $\widehat{G}(\xi)<1$ for all $\xi \neq 0$. In order to see that, write

$$
\widehat{G}(\xi)=\int_{\mathbb{R}^{d}} \prod_{k=1}^{d} e^{-2 \pi i x_{k} \xi_{k}} G(x) d x=\int_{\mathbb{R}^{d}} \prod_{k=1}^{d}\left(\cos \left(2 \pi x_{k} \xi_{k}\right)-i \sin \left(2 \pi x_{k} \xi_{k}\right)\right) G(x) d x,
$$

so that $G$ being even easily implies that only real valued contributions survive in the above integral; such real valued contributions are of the form

$$
\int_{\mathbb{R}^{d}} f_{h, k}(x, \xi) G(x) d x
$$

where the functions $f_{h, k}$ are such that $\left|f_{h, k}(x, \xi)\right| \leq 1$ and $\left|f_{h, k}(x, \xi)\right|<1$ for $x$ ranging on a set of positive measure. Therefore, we have proven that

$$
\widehat{\rho_{\infty}}(\xi)=0 \text { for all } \xi \neq 0
$$

and $\widehat{\rho_{\infty}}(0)=1$. This implies that $\rho(x)=0$ almost everywhere, which contradicts the fact that $\rho$ has unit mass.

3.3. Stationary solutions for $\varepsilon<1$. Let us now provide a minimizer for the entropy functional in the case $\varepsilon<1$, which implies the existence of a nontrivial $L^{2} \cap \mathcal{P}$ steady state for (3.1) in view of Proposition 3.1. Such a result is proven rigorously in [3, Theorem 1], which we recall here.

TheOREm 3.6 (Existence of minimizers, [3]). Let $\varepsilon<1$. Then, there exists a radially symmetric non-increasing minimizer $\rho \in \mathcal{P} \cap L^{2}\left(\mathbb{R}^{d}\right)$ for the entropy functional $E$ restricted to $\mathcal{P}$ with $\rho \neq 0$. 
We refer the reader to [3] for the details of the proof, which is based on a sort of subadditivity property needed to provide suitable compactness of the minimizing sequence; cf. [3, Lemma 2]. For the sake of clarity, we shall still provide the simple proof of the fact that global minima of $E$ under mass constraint are strictly negative, which forces the minimizer to be nonzero.

Lemma 3.1. Let $\varepsilon<1$. Then $\inf _{\rho \in \mathcal{P} \cap L^{2}\left(\mathbb{R}^{d}\right)} E[\rho]<0$.

Proof. We consider the family $\sigma_{\lambda} \in L^{2} \cap \mathcal{P}$, where

$$
\sigma_{\lambda}(x)=\frac{1}{2 \lambda} \chi_{[-\lambda, \lambda]}(x) .
$$

For $\varepsilon<1$ we have

$$
\begin{aligned}
E\left[\sigma_{\lambda}\right] & =\frac{\varepsilon}{4 \lambda}-\frac{1}{8 \lambda^{2}} \int_{-\lambda}^{\lambda} \int_{-\lambda}^{\lambda} G(x-y) d y d x=\frac{\varepsilon}{4 \lambda}-\frac{1}{4 \lambda} \int_{-\lambda}^{\lambda} G(z) d z \\
& =\frac{\varepsilon}{4 \lambda}-\frac{1}{8 \lambda^{2}} \int_{-\lambda}^{\lambda}(F(\lambda-u)-F(-\lambda-u)) d u,
\end{aligned}
$$

with $F(u)=\int_{-\infty}^{u} G(\xi) d \xi$. Since

$$
\begin{aligned}
& \lim _{\lambda \rightarrow+\infty} \frac{1}{2 \lambda} \int_{-\lambda}^{\lambda}(F(\lambda-u)-F(-\lambda-u)) d u \\
= & \lim _{\lambda \rightarrow+\infty} \frac{1}{2 \lambda}\left(\int_{0}^{2 \lambda} d z F(z)-\int_{-2 \lambda}^{0} F(z) d z\right) \\
= & \lim _{\lambda \rightarrow+\infty}(F(2 \lambda)-F(-2 \lambda))=1,
\end{aligned}
$$

we easily obtain that there exists a $\bar{\lambda}$ such that $E\left[\sigma_{\bar{\lambda}}\right]<0$.

\section{Stationary solutions in the $1 d$ case}

In this section we prove the main result of our paper, namely that nontrivial stationary solutions (which always exist in the case $\varepsilon<1$ ) in one space dimension with fixed mass and center of mass are unique. We shall first provide certain necessary conditions on the steady states and then prove that they are unique under such conditions. The main tool in this procedure is the use of the strong version of the Krein-Rutman Theorem 4.10.

We start with a necessary condition on the steady states which deals with a property of their support.

LEmma 4.1 (Steady states have connected support). Let $\rho$ be a stationary solution to (3.1) in one space dimension, namely

$$
\rho \partial_{x}(\varepsilon \rho-G * \rho)=0 \quad \text { a.e. on } \mathbb{R} .
$$

Then, $\operatorname{supp}(\rho)$ is a connected set.

Proof. Let $\rho$ solve (4.1). Let us first assume that $\rho$ is compactly supported. Suppose that $\operatorname{supp}(\rho)$ is not connected. Accordingly, let $[a, b]$ be a non trivial interval such that

$$
\begin{array}{ll}
\rho(x) \not \equiv 0, & \text { if } x<a, \\
\rho(x)=0, & \text { if } a \leq x \leq b, \\
\rho(x) \not \equiv 0, & \text { if } x>b .
\end{array}
$$


Let us introduce the velocity field

$$
V(x):=\left\{\begin{array}{lll}
-1 & \text { if } & x \in(-\infty, a) \\
1 & \text { if } & x \in(b,+\infty)
\end{array}\right.
$$

and let $V \in C^{1}(\mathbb{R})$. Let $u(x, s)$ be a local solution to the Cauchy problem for the continuity equation

$$
\left\{\begin{array}{l}
\partial_{s} u+\partial_{x}(u V)=0 \\
u(x, 0)=\rho(x)
\end{array}\right.
$$

Let us compute the evolution of the energy $E$ along $u$ at the time $s=0$ :

$$
\left.\frac{d}{d s} E[u(s)]\right|_{s=0}=\left.\int u_{s}(\varepsilon u(x, s)-G * u(x, s)) d x\right|_{s=0}=\int \rho V \partial_{x}(\varepsilon \rho-G * \rho) d x=0 .
$$

Then, by the definition of $V$ we have

$$
\varepsilon \int_{\mathbb{R}} \rho V \partial_{x} \rho=\frac{\varepsilon}{2} \int_{-\infty}^{a} \partial_{x} \rho^{2} d x+\frac{\varepsilon}{2} \int_{a}^{b} V \partial_{x} \rho^{2} d x-\frac{\varepsilon}{2} \int_{b}^{+\infty} \partial_{x} \rho^{2} d x=0
$$

because $\rho_{x}=0$ on $[a, b]$ and $\rho=0$ on $x=a, b$ and at $\pm \infty$. Hence, we have

$$
0=\int_{-\infty}^{+\infty} \rho V \partial_{x} G * \rho d x=-\int_{-\infty}^{a} \rho G^{\prime} * \rho d x+\int_{b}^{+\infty} \rho G^{\prime} * \rho d x
$$

We compute

$$
\begin{aligned}
& \int_{-\infty}^{a} \rho G^{\prime} * \rho d x \\
= & \int_{-\infty}^{a} \int_{-\infty}^{a} \rho(x) G^{\prime}(x-y) \rho(y) d y d x+\int_{-\infty}^{a} \int_{b}^{+\infty} \rho(x) G^{\prime}(x-y) \rho(y) d y d x,
\end{aligned}
$$

and note that the first term on the above right-hand side is zero since $G^{\prime}$ is odd and the integration domain is symmetric in $x$ and $y$. Since $G^{\prime}(z) \geq 0$ as $z \leq 0$, we have for the second term

$$
\rho(x) G^{\prime}(x-y) \rho(y) \geq 0 \quad \text { on }(x, y) \in(-\infty, a) \times(b,+\infty) .
$$

In a similar way one can prove that

$$
\int_{b}^{+\infty} \rho G^{\prime} * \rho d x=\int_{b}^{+\infty} d x \int_{-\infty}^{a} d y \rho(x) G^{\prime}(x-y) \rho(y),
$$

with the integrand $\rho(x) G^{\prime}(x-y) \rho(y) \leq 0$ on the integration domain. Therefore, (4.4) implies that

$$
\rho(x) \rho(y) \equiv 0 \quad \text { on } \quad\{x<a\} \cap\{y>b\} .
$$

We have thus proven that, whenever (4.2) holds, then (4.5) must necessarily be satisfied. Let $A, B$ be two nonempty connected components of $\operatorname{supp}(\rho)$ and let 
$[\alpha, \beta]$ be the maximal interval such that

$$
\begin{array}{ll}
a<b, & \text { for all } a \in A, b \in B, \\
\rho \equiv 0, & \text { on }[\alpha, \beta], \\
\alpha \geq a, & \text { for all } a \in A, \\
\beta \leq b, & \text { for all } b \in B .
\end{array}
$$

Then, $\rho(x) \rho(y)=0$ for all $(x, y)$ such that

$$
x<\alpha, \quad y>\beta,
$$

which implies that either $A$ or $B$ cannot be in the support of $\rho$, and that is a contradiction. In order to generalize the proof to a stationary solution $\rho$ which is not compactly supported, one can cutoff $\rho$ to have compact support in such a way that the $L^{2}$ norm of the compactly supported approximation is arbitrarily close to the $L^{2}$ norm of $\rho$. Then, the estimate

$$
\varepsilon \int\left|\rho \partial_{x} \rho\right| d x \leq\|G\|_{L^{1}}\|\rho\|_{L^{2}}^{2}
$$

implies that the integrals in (4.3) converge at infinity, therefore all the above computations are valid up to an arbitrary difference which vanishes in the limit.

REMARK 4.2. In the case $\operatorname{supp}(G)=[-R, R]$ one can use the same strategy as in Lemma 4.1 to prove that, given two connected components $A, B$ of $\operatorname{supp}(\rho)$, one has $\operatorname{dist}(A, B)>2 R$. The proof is a straightforward generalization of the above arguments, and it is therefore left to the reader.

We now exploit a standard symmetric rearrangement technique to prove that the minimizers of the energy are symmetric and monotonically decreasing on $x>0$ under the constraint of zero center of mass; cf. [47, 48].

Proposition 4.3. Let $\rho_{\infty}$ be a minimizer for the energy

$$
E[\rho]=\frac{\varepsilon}{2} \int \rho^{2}(x) d x-\frac{1}{2} \iint G(x-y) \rho(x) \rho(y) d y d x
$$

under the constraint that the center of mass is zero. Then, $\rho_{\infty}$ is symmetric and monotonically decreasing on $x>0$.

Proof. We have to prove that the energy strictly decreases when a function $u$ which is not symmetric and decreasing on $x>0$ is replaced by its symmetric rearrangement

$$
u^{*}(x)=\sup \{t \geq 0: \operatorname{meas}(\{u>t\})>2|x|\} .
$$

For every exponent $p \geq 1$ the following holds:

$$
\int_{\mathbb{R}}\left(u^{*}\right)^{p} d x=\int_{\mathbb{R}}(u)^{p} d x
$$

therefore the $L^{2}$ part of the energy is invariant when passing from $u$ to $u^{*}$. As for the interaction energy, we recall the well known Riesz's rearrangement inequality (see e.g. [48]),

$$
\int_{\mathbb{R}^{d}} \int_{\mathbb{R}^{d}} f(x) g(x-y) h(y) d y d x \leq \int_{\mathbb{R}^{d}} \int_{\mathbb{R}^{d}} f^{*}(x) g^{*}(x-y) h^{*}(y) d y d x,
$$


which holds for all nonnegative functions $f, g, h$ vanishing at infinity. Moreover, if $g$ is strictly decreasing on $x>0$ and symmetric, then equality in (4.8) holds if and only if $f(x)=f^{*}\left(x-x_{0}\right)$ and $h(x)=h^{*}\left(x-x_{0}\right)$ for some common $x_{0}$. Apply such a theorem to our case, using $G^{*}=G$ and the fact that $u$ is not symmetric up to translations.

Let us rephrase Lemma 3.3 in the one-dimensional case.

Lemma 4.4. Let $\rho$ be a $1 d$ steady state, i.e.

$$
\varepsilon \rho=G * \rho+C \quad \text { on } \operatorname{supp}[\rho]
$$

for some $C \in \mathbb{R}$. Then, $C=2 E[\rho]$.

Proof. The support of $\rho$ is connected in view of Lemma 4.1, and therefore Lemma 3.3 applies.

Lemma 4.5. Let $\rho \in \mathcal{P} \cap L^{2}$ and let $x_{0} \in \mathbb{R}$. Let $\rho_{x_{0}}$ be defined by

$$
\rho_{x_{0}}(x):=\rho\left(x+x_{0}\right) \text {. }
$$

Then, $E\left[\rho_{x_{0}}\right]=E[\rho]$.

Proof. It follows by direct computation of the energy and by a change of variable under the integral sign.

LEMma 4.6. Let $\rho$ be a steady state with $\varepsilon<1$. Then, the support of $\rho$ is compact.

Proof. We know from Lemma 4.1 that the support of $\rho$ is a connected set. Suppose that $\operatorname{supp}(\rho)$ is not bounded. That means that $\operatorname{supp}(\rho)$ is of the form $(-\infty, b)(b$ possibly $+\infty)$ or $(a,+\infty)(a$ possibly $-\infty)$. Assume first $\operatorname{supp}(\rho)=$ $(a,+\infty)$. Then, Lemma 4.4 implies

$$
2 E[\rho]=\varepsilon \rho(x)-\int_{a}^{+\infty} G(x-y) \rho(y) d y=0
$$

for all $x \in(a,+\infty)$. Now, there are two possibilities: either $a=-\infty$ or $a>-\infty$. In the latter case, evaluation on $x=a$ implies

$$
0=\varepsilon \rho(a)=\int_{a}^{+\infty} G(a-y) \rho(y) d y
$$

which is a contradiction because the integral on the right hand side is strictly positive in view of $\operatorname{supp}(G)=\mathbb{R}$. In the former case $a=-\infty$ and we have $\operatorname{supp}(\rho)=\mathbb{R}$, which implies

$$
\varepsilon \rho(x)=\int_{-\infty}^{+\infty} G(x-y) \rho(y) d y
$$

for all $x \in \mathbb{R}$. We can therefore integrate over $\mathbb{R}$ to obtain

$$
\varepsilon=\|G\|_{L^{1}}=1,
$$

which is a contradiction. The same proof can be produced in the case $\operatorname{supp}(\rho)=$ $(-\infty, b)$. Therefore, the support of $\rho$ can only be a bounded interval. 
Lemma 4.7. Let $\rho$ be a steady state. Then there exists a symmetric steady state $\widetilde{\rho}$ such that

$$
E[\widetilde{\rho}]=E[\rho] .
$$

Proof. From Lemma 4.1 and Lemma 4.6 we know that $\operatorname{supp}[\rho]=(a, b)$ for some $a, b \in \mathbb{R}$. For a given $x \in(a, b)$ we have

$$
\varepsilon \rho(x)=G * \rho(x)+C
$$

for some $C \in \mathbb{R}$. Evaluation on $x=a$ and $x=b$ gives

$$
C=-\int_{a}^{b} G(a-y) \rho(y) d y=-\int_{a}^{b} G(b-y) \rho(y) d y .
$$

Let $\bar{\rho}(x)=\rho\left(x+x_{0}\right)$ with $x_{0}=(a+b) / 2$. Then $\bar{\rho}$ is still a steady state and it satisfies $E[\bar{\rho}]=E[\rho]$ thanks to Lemma 4.5. Moreover, the support of $\bar{\rho}$ is symmetric. Let us introduce

$$
\widetilde{\rho}(x):=\frac{1}{2}(\bar{\rho}(x)+\bar{\rho}(-x)) .
$$

Clearly, $\operatorname{supp}[\widetilde{\rho}]=\operatorname{supp}[\bar{\rho}]$ and we have, for all $x \in \operatorname{supp}[\widetilde{\rho}]$,

$$
\begin{aligned}
& \varepsilon \widetilde{\rho}(x)=\frac{\varepsilon}{2}(\bar{\rho}(x)+\bar{\rho}(-x))=\frac{\varepsilon}{2}\left(\rho\left(x+x_{0}\right)+\rho\left(-x+x_{0}\right)\right) \\
= & \frac{1}{2} \int_{a}^{b} G\left(x+x_{0}-y\right) \rho(y) d y+\frac{1}{2} \int_{a}^{b} G\left(-x+x_{0}-y\right) \rho(y) d y+C \\
= & \frac{1}{2} \int_{(a-b) / 2}^{(b-a) / 2} G(x-z) \bar{\rho}(z) d y+\frac{1}{2} \int_{(a-b) / 2}^{(b-a) / 2} G(-x-z) \bar{\rho}(z) d y+C \\
= & \frac{1}{2} \int_{(a-b) / 2}^{(b-a) / 2} G(x-z) \bar{\rho}(z) d y+\frac{1}{2} \int_{(a-b) / 2}^{(b-a) / 2} G(x-z) \bar{\rho}(-z) d y+C \\
= & \int_{(a-b) / 2}^{(b-a) / 2} G(x-z) \frac{1}{2}(\bar{\rho}(z)+\bar{\rho}(-z)) d z+C=\int_{(a-b) / 2}^{(b-a) / 2} G(x-z) \widetilde{\rho}(z) d z+C,
\end{aligned}
$$

where we have used the symmetry of $G$. The above computation shows that $\tilde{\rho}$ has the same energy as $\rho$ in view of the results in Lemma 4.5 and Lemma 4.4. $\square$ Lemma 4.8 (Support of a minimizer). Let $\rho_{\infty}$ be a global minimizer of E. Let $\rho$ be a steady state such that

$$
\operatorname{meas}\left(\operatorname{supp}\left[\rho_{\infty}\right]\right) \leq \operatorname{meas}(\operatorname{supp}[\rho]) .
$$

Then $\rho$ is also a minimizer.

Proof. Assume first that we are in the special case $\operatorname{supp}\left[\rho_{\infty}\right] \subseteq \operatorname{supp}[\rho]$. Let us compute the second variation of $E$ around the minimizer $\rho_{\infty}$ along the direction $\rho_{\infty}-\rho$ :

$$
\begin{aligned}
& \left.\frac{d^{2}}{d \delta^{2}} E\left[\rho_{\infty}+\delta\left(\rho-\rho_{\infty}\right)\right]\right|_{\delta=0} \\
= & \varepsilon \int\left(\rho-\rho_{\infty}\right)^{2} d x-\iint G(x-y)\left(\rho(x)-\rho_{\infty}(x)\right)\left(\rho(y)-\rho_{\infty}(y)\right) d y d x \\
= & 2 E[\rho]+2 E\left[\rho_{\infty}\right]-2 \int_{\operatorname{supp}\left[\rho_{\infty}\right]} \rho_{\infty}(\varepsilon \rho-G * \rho) d x \\
= & 2 E[\rho]+2 E\left[\rho_{\infty}\right]-4 E[\rho],
\end{aligned}
$$


where the last step is justified by the fact that $\operatorname{supp}[\rho] \subseteq \operatorname{supp}\left[\rho_{\infty}\right]$. Therefore, since $\rho_{\infty}$ is a minimizer, the second derivative above is nonnegative, i.e.

$$
0 \leq\left.\frac{d^{2}}{d \delta^{2}} E\left[\rho_{\infty}+\delta\left(\rho-\rho_{\infty}\right)\right]\right|_{\delta=0}=2\left(E\left[\rho_{\infty}\right]-E[\rho]\right),
$$

which yields $E[\rho] \leq E\left[\rho_{\infty}\right]$. Since $\rho_{\infty}$ is a minimizer, then so is $\rho$. In the general case in which $\operatorname{supp}\left[\rho_{\infty}\right] \nsubseteq \operatorname{supp}[\rho]$, consider a translation $\rho_{x_{0}}(x)=\rho\left(x-x_{0}\right)$ in such a way that the support of $\rho$ contains the support of $\rho_{\infty}$. Since the energy is invariant after translation in view of Lemma 4.5, the assertion is proven.

We are now getting closer to the proof of our uniqueness result. Let us recall the following important theorems; see e.g. [28] and the references therein.

Theorem 4.9 (Krein-Rutman Theorem). Let $X$ be a Banach space, let $K \subset$ $X$ be a total cone, i.e. such that $\lambda K \subset K$ for all $\lambda \geq 0$ and such that the set $\{u-v, u, v \in K\}$ is dense in $X$. Let $T$ be a compact linear operator such that $T(K) \subset K$ with positive spectral radius $r(T)$. Then $r(T)$ is an eigenvalue for $T$ with an eigenvector $u \in K \backslash\{0\}$.

An important consequence [28] of the Krein-Rutman Theorem, which will be extremely useful in the sequel, is the following.

TheOREM 4.10 (Krein-Rutman Theorem, strong version). Let $X$ be a Banach space, $K \subset X$ a solid cone, i.e. such that $\lambda K \subset K$ for all $\lambda \geq 0$ and such that $K$ has a nonempty interior $K_{0}$. Let $T$ be a compact linear operator which is strongly positive with respect to $K$, i.e. such that $T[u] \in K_{0}$ if $u \in K$. Then

(i) The spectral radius $r(T)$ is strictly positive and $r(T)$ is a simple eigenvalue with an eigenvector $v \in K_{0}$. There is no other eigenvalue with a corresponding eigenvector $v \in K$.

(ii) $|\lambda|<r(T)$ for all other eigenvalues $\lambda \neq r(T)$.

We shall now prove the uniqueness of symmetric steady states with unit mass which are monotonically decreasing on the positive semi-axis in the case $\varepsilon<1$. We already know that under the above assumptions we can write, for $x \in[-L, L]=\operatorname{supp}[\rho]$,

$$
\varepsilon \rho(x)=\int_{-L}^{L} G(x-y) \rho(y) d y+C, \quad C=2 E[\rho] .
$$

Taking the derivative with respect to $x \in[-L, L]$ we obtain

$$
\varepsilon \rho^{\prime}(x)=\frac{d}{d x} \int_{-L}^{L} G(x-y) \rho(y) d y=\frac{d}{d x} G * \rho(x)=\int_{-L}^{L} G(x-y) \rho^{\prime}(y) d y .
$$

The symmetry of $\rho$ and $G$ implies, for $x \in[0, L]$,

$$
\begin{aligned}
& \varepsilon \rho^{\prime}(x)=-\int_{0}^{L} G(x+y) \rho^{\prime}(y) d y+\int_{0}^{L} G(x-y) \rho^{\prime}(y) d y \\
= & \int_{0}^{L}[G(x-y)-G(x+y)] \rho^{\prime}(y) d y .
\end{aligned}
$$


Assuming that $\rho \in C^{1}([-L, L])$, finding a steady state with the above assumptions is equivalent to finding $\rho$ on $[0, L]$ such that

$$
\begin{aligned}
& \rho(L)=0, \\
& -\rho^{\prime}(x)=u(x), \quad x \in[0, L], \\
& u \geq 0, \quad \text { and } u \text { solves } \quad \varepsilon u(x)=\int_{0}^{L} H(x, y) u(y) d y, \\
& H(x, y)=G(x-y)-G(x+y) .
\end{aligned}
$$

To convince ourselves that this is true, integrate

$$
-\varepsilon \rho^{\prime}(x)=-\int_{0}^{L}(G(x-y)-G(x+y)) \rho^{\prime}(y) d y
$$

over the interval $[\xi, L]$ for some $\xi \in[0, L)$. Then integration by parts and the fact that $\rho(L)=0$ imply

$$
\begin{aligned}
-\varepsilon \rho(\xi)= & -\int_{\xi}^{L} d x \int_{0}^{L}(G(x-y)-G(x+y)) \rho^{\prime}(y) d y \\
= & -\int_{\xi}^{L} d x[(G(x-L)-G(x+L)) \rho(L)-(G(x)-G(x)) \rho(0)] \\
& \quad+\int_{\xi}^{L} d x \int_{0}^{L}\left(-G^{\prime}(x-y)-G^{\prime}(x+y)\right) \rho(y) d y \\
= & \int_{0}^{L} \rho(y) d y \int_{\xi}^{L}\left(-G^{\prime}(x-y)-G^{\prime}(x+y)\right) d x \\
= & \int_{0}^{L} \rho(y)[-G(L-y)-G(L+y)+G(\xi-y)+G(\xi+y)],
\end{aligned}
$$

which implies, by the symmetry of $G$,

$$
\varepsilon \rho(x)=\int_{-L}^{L} G(x-y) \rho(y) d y+C, \quad C=-\int_{0}^{L}(G(L-y)+G(L+y)) \rho(y) d y .
$$

For further reference, we introduce the operator

$$
\mathcal{G}_{L}[\rho](x):=\int_{0}^{L}[G(x-y)+G(x+y)-G(L-y)-G(L+y)] \rho(y) d y
$$

on the Banach space

$$
Y_{L}:=\{\rho \in C([0, L]): \rho(L)=0\} .
$$

In order to simplify the notation, we also define the following operator:

$$
\mathcal{H}_{L}[u](x):=\int_{0}^{L} H(x, y) u(y) d y=\int_{0}^{L}(G(x-y)-G(x+y)) u(y) d y .
$$

Proposition 4.11. For a fixed $L>0$ there exists a unique symmetric function $\rho \in C^{2}([-L, L])$ with unit mass and with $\rho^{\prime}(x) \leq 0$ on $x \geq 0$ such that $\rho$ solves 
(4.10) for some $\varepsilon=\varepsilon(L)>0$. Such a function $\rho$ also satisfies $\rho^{\prime \prime}(0)<0$. Moreover, $\varepsilon(L)$ is the largest eigenvalue of the compact operator $\mathcal{G}_{L}$ on the space Banach $Y_{L}$ and any other eigenfunction of $\mathcal{G}_{L}$ on $Y_{L}$ with unit mass has the corresponding eigenvalue $\varepsilon^{\prime}$ satisfying $\left|\varepsilon^{\prime}\right|<\varepsilon(L)$.

Proof. Since $G$ is decreasing on the half-line $[0,+\infty)$ we get

$$
H(x, y)=G(x-y)-G(x+y) \geq 0, \quad \text { on } \quad x, y \geq 0 .
$$

Consider now the Banach space

$$
X_{L}=\left\{f \in C^{1}([0, L]): f(0)=0\right\}
$$

endowed with the $C^{1}$ norm

$$
\|f\|_{X_{L}}=\|f\|_{L^{\infty}([0, L])}+\left\|f^{\prime}\right\|_{L^{\infty}([0, L])} .
$$

It can be easily seen that the set

$$
K_{L}:=\left\{f \in X_{L}: f \geq 0\right\}
$$

is a solid cone in $X_{L}$. Indeed, let us define the subset of $K_{L}$

$$
H:=\left\{f \in K: f^{\prime}(0)>0, \quad f(x)>0 \text { for all } x>0\right\} .
$$

Then it is easily seen that $H \subset K_{L}^{\circ}$, where the latter denotes the interior of $K$. Now, for a given $u \in K$, we have

$$
\mathcal{H}_{L}[u](x)=\int_{0}^{L} H(x, y) u(y) d y \geq 0
$$

for all $x \in[0, L]$, and

$$
\mathcal{H}_{L}[u](0)=\int_{0}^{L} H(0, y) u(y) d y=\int_{0}^{L}(G(-y)-G(y)) u(y) d y=0 .
$$

Therefore $\mathcal{H}_{L}$ is a positive operator in the sense provided by the definition of the cone $K$. Now, we aim to prove that $\mathcal{H}_{L}$ is strongly positive, i.e. for a given $u \in K, \mathcal{H}[u] \in K^{\circ}$. In order to see that, for a $u \in K \backslash\{0\}$ compute

$$
\left(\mathcal{H}_{L}[u]\right)^{\prime}(0)=\int_{0}^{L}\left(G^{\prime}(-y)-G^{\prime}(y)\right) u(y) d y=-2 \int_{0}^{L} G^{\prime}(y) u(y) d y>0 .
$$

Moreover, for $x>0$ we have

$$
\mathcal{H}_{L}[u](x)=\int_{0}^{L} H(x, y) u(y) d y=\int_{0}^{L}(G(x-y)-G(x+y)) u(y) d y>0
$$

because the function $G(x-y)-G(x+y)$ is strictly positive on $y \in(0, L]$ and $u$ is nonnegative on the same interval. Therefore $\mathcal{H}_{L}[u] \in H \subset K^{\circ}$. Hence, we can apply the stronger version of the Krein-Rutman Theorem 4.10, which implies the existence of a simple eigenvalue $\varepsilon>0$ equal to the spectral radius of $\mathcal{H}_{L}$. More precisely, there exists a family of solutions $u$ to

$$
\varepsilon u=\mathcal{H}_{L}[u]
$$


generated by one given nontrivial element $\bar{u}$ in the interior of $K$. This implies that the corresponding set of symmetric and monotone $\rho$ solving (4.10) satisfies

$$
\rho(x)=\rho(x)-\rho(L)=-\int_{x}^{L} \rho^{\prime}(y) d y=\int_{x}^{L} u(y) d y=\alpha \int_{x}^{L} \bar{u}(y) d y,
$$

for $\alpha>0$. We choose $\alpha$ as

$$
\alpha=\left(2 \int_{0}^{L} \int_{x}^{L} \bar{u}(y) d y d x\right)^{-1},
$$

and we obtain that $\rho$ has unit mass on $[-L, L]$. It is clear that $\rho^{\prime}(x) \leq 0$ for $x \geq 0$, $\rho^{\prime}(0)=0$, and $\rho^{\prime \prime}(0)<0$. In view of the statement (i) of Theorem 4.10, there exist no other eigenvalues to $\mathcal{H}_{L}$ with eigenvectors in $K_{L}$ besides the one with eigenfunction $\bar{u}$, and all other eigenvalues $\varepsilon^{\prime}$ with eigenfunctions in $X_{L}$ satisfy $\left|\varepsilon^{\prime}\right|<\varepsilon$.

The eigenvalue $\varepsilon$ (which coincides with the spectral radius of $\mathcal{H}_{L}$ ) can be considered as a function of $L$, namely $\varepsilon=\varepsilon(L)$. The behavior of such a function is established in the next proposition. From now on we shall denote by $u_{L}=-\frac{d}{d x} \rho_{L}$ the unique eigenfunction of $\mathcal{H}_{L}$ on $K_{L}$ satisfying

$$
2 \int_{0}^{L} \rho_{L}(x) d x=-2 \int_{0}^{L} \int_{x}^{L} u(y) d y d x=1 .
$$

The next proposition shows a key property needed in order to prove our uniqueness result. Roughly speaking, the result in Proposition 4.11 provides uniqueness for a given fixed $L$, whereas our aim is to achieve uniqueness for a fixed $\varepsilon$. To perform this task, we have to prove that the function $(0,+\infty) \ni L \mapsto$ $\varepsilon=\varepsilon(L) \in(0,1)$ is a continuous bijection.

Proposition 4.12 (Behavior of the function $\varepsilon(L)$ ). The simple eigenvalue $\varepsilon(L)$ found in Proposition 4.11 is uniquely determined as a function of $L$ with the following properties:

(i) $\varepsilon(L)$ is continuous and strictly increasing with respect to $L$;

(ii) $\lim _{L \rightarrow+\infty} \varepsilon(L)=1$;

(iii) $\varepsilon(0)=0$.

Proof. Step 1: Monotonicity of $\varepsilon(L)$. Let us consider the equation

$$
\varepsilon(L) u_{L}(x)=\mathcal{H}_{L}\left[u_{L}\right](x)=\int_{0}^{L} H(x, y) u_{L}(y) d y, \quad x \in[0, L],
$$

where $u_{L}$ is the unique eigenfunction obtained in Proposition 4.11 satisfying (4.13). We multiply the above equation by $u_{L}(x)$ and integrate over $[0, L]$ to obtain

$$
\varepsilon(L) \int_{0}^{L} u_{L}(x)^{2} d x=\int_{0}^{L} \mathcal{H}_{L}\left[u_{L}\right](x) u(x) d x .
$$

Recall that the eigenvalue $u_{L}$ satisfies $u_{L}(0)=0$ and, for $x \in(0, L]$,

$$
u_{L}(x)=\frac{1}{\varepsilon(L)} \int_{0}^{L} H(x, y) u(y) d y>0
$$


since $H(x, y)=G(x-y)-G(x+y)>0$ for all $y \in[0, L]$ under the assumption $x>$ 0 in view of the strict decreasing monotonicity of $G$ on $x>0$, For a general $L \in(0,+\infty)$ and a $\delta>0$ (small enough) we have

$$
\begin{aligned}
I_{1} & :=\varepsilon(L+\delta) \int_{0}^{L+\delta} u_{L+\delta}^{2}(x) d x-\varepsilon(L) \int_{0}^{L} u_{L}^{2}(x) d x \\
& =\int_{0}^{L+\delta} \mathcal{H}_{L+\delta}\left[u_{L+\delta}\right](x) u_{L+\delta}(x) d x-\int_{0}^{L} \mathcal{H}_{L}\left[u_{L}\right](x) u_{L}(x) d x=: I_{2} .
\end{aligned}
$$

We shall now analyze the two terms $I_{1}$ and $I_{2}$ separately. Let us first extend $u_{L}$ on $(L, L+\delta]$ as follows:

$$
\bar{u}_{L}(x)= \begin{cases}u_{L}(x) & \text { if } x \in[0, L], \\ 0 & \text { if } x \in(L, L+\delta] .\end{cases}
$$

In view of the above definition, we have $\mathcal{H}_{L+\delta}\left[\bar{u}_{L}\right](x)=\mathcal{H}_{L}\left[\bar{u}_{L}\right](x)$ on $x \in[0, L]$, and

$$
\mathcal{H}_{L+\delta}\left[\bar{u}_{L}\right](x)= \begin{cases}\varepsilon(L) \bar{u}_{L}(x) & \text { if } x \in[0, L], \\ \int_{0}^{L} H(x, y) u_{L}(y) d y & \text { if } x \in(L, L+\delta] .\end{cases}
$$

The term $I_{1}$ can be expanded as follows:

$$
\begin{aligned}
I_{1}=(\varepsilon & (L+\delta)-\varepsilon(L)) \int_{0}^{L+\delta} u_{L+\delta}^{2}(x) d x \\
& +\varepsilon(L) \int_{0}^{L+\delta}\left(u_{L+\delta}(x)-\bar{u}_{L}(x)\right)\left(u_{L+\delta}(x)+\bar{u}_{L}(x)\right) d x .
\end{aligned}
$$

$I_{2}$ is given by

$$
\begin{aligned}
I_{2}= & \int_{0}^{L+\delta}\left(\mathcal{H}_{L+\delta}\left[u_{L+\delta}\right] u_{L+\delta}-\mathcal{H}_{L+\delta}\left[\bar{u}_{L}\right] \bar{u}_{L}\right) d x \\
= & \int_{0}^{L+\delta}\left(\mathcal{H}_{L+\delta}\left[u_{L+\delta}\right](x)-\mathcal{H}_{L+\delta}\left[\bar{u}_{L}\right](x)\right) u_{L+\delta}(x) d x \\
& \quad+\int_{0}^{L+\delta} \mathcal{H}_{L+\delta}\left[\bar{u}_{L}\right](x)\left(u_{L+\delta}(x)-\bar{u}_{L}(x)\right) d x \\
= & \int_{0}^{L+\delta}\left(\mathcal{H}_{L+\delta}\left[u_{L+\delta}\right](x)-\mathcal{H}_{L+\delta}\left[\bar{u}_{L}\right](x)\right) u_{L+\delta}(x) d x \\
& \quad+\varepsilon(L) \int_{0}^{L+\delta} \bar{u}_{L}(x)\left(u_{L+\delta}(x)-\bar{u}_{L}(x)\right) d x \\
& \quad+\int_{L}^{L+\delta} d x \int_{0}^{L} d y H(x, y) \bar{u}_{L}(y) u_{L+\delta}(x),
\end{aligned}
$$

where we have used (twice) $\bar{u}_{L}(x)=0$ on $x \in(L, L+\delta]$. Therefore, on substituting $I_{1}$ and $I_{2}$ in (4.14) we can cancel some terms and use the eigenvalue property 


$$
\begin{aligned}
& \mathcal{H}_{L+\delta}\left[u_{L+\delta}\right]=\varepsilon(L+\delta) u_{L+\delta} \text { to obtain } \\
& \quad-\varepsilon(L) \int_{0}^{L+\delta} \bar{u}_{L}(x) u_{L+\delta}(x) d x \\
& =\int_{L}^{L+\delta} d x \int_{0}^{L} d y H(x, y) \bar{u}_{L}(y) u_{L+\delta}(x)-\int_{0}^{L+\delta} d x \int_{0}^{L+\delta} d y H(x, y) \bar{u}_{L}(y) u_{L+\delta}(x) \\
& =\int_{L}^{L+\delta} d x \int_{0}^{L} d y H(x, y) \bar{u}_{L}(y) u_{L+\delta}(x)-\varepsilon(L+\delta) \int_{0}^{L+\delta} u_{L+\delta}(y) \bar{u}_{L}(y) d y,
\end{aligned}
$$

where we have used the definition of $\mathcal{H}_{L+\delta}$, and the property $H(x, y)=H(y, x)$. Therefore, we have

$$
(\varepsilon(L+\delta)-\varepsilon(L)) \int_{0}^{L} u_{L}(x) u_{L+\delta}(x) d x=\int_{L}^{L+\delta} d x \int_{0}^{L} d y H(x, y) u_{L}(y) u_{L+\delta}(x),
$$

and the positivity property of $u_{L}$ implies that

$$
\varepsilon(L+\delta)>\varepsilon(L),
$$

which proves the monotonicity of $\varepsilon(L)$. Notice that formula (4.15) is totally independent from the way we extended $u_{L}$ on $[L, L+\delta]$. We shall now use the formula (4.15) to prove continuity of $\varepsilon$ with respect to $L$. In order to perform this task, we shall work in the following two steps.

Step 2: Local uniform bound For $L \mapsto\left\|u_{L}\right\|_{L^{\infty}([0, L])}$. Let $L_{0} \in(0,+\infty)$ be fixed, we aim to prove that there exists a neighborhood $I_{0}=\left[L_{0}-\delta, L_{0}+\delta\right]$ of $L_{0}$ such that $\sup _{L \in I_{0}}\left\|u_{L}\right\|_{L^{\infty}([0, L])}$ is finite. In order to see that, choose $\delta<L_{0} / 2$ so that the eigenvalue property of $u_{L}$ and the monotonicity of $\varepsilon(L)$ imply

$$
u_{L}(x)<\frac{1}{\varepsilon\left(L_{0} / 2\right)} \int_{0}^{L} H(x, y) u_{L}(y) d y \leq \frac{1}{\varepsilon\left(L_{0} / 2\right)}\|G\|_{L^{\infty}} \int_{0}^{L} u_{L}(y) d y .
$$

Recall that $\varepsilon(L) \rho_{L}=\mathcal{G}_{L}\left[\rho_{L}\right]$ implies

$$
\begin{aligned}
\int_{0}^{L} u_{L}(y) d y=-\rho_{L}(L)+\rho_{L}(0) & =\rho_{L}(0) \\
& =\frac{1}{\varepsilon(L)} \int_{0}^{L}(2 G(y)-G(L-y)-G(L+y)) \rho_{L}(y) d y \\
& \leq \frac{\|G\|_{L^{\infty}}}{\varepsilon\left(L_{0} / 2\right)} \int_{-L}^{L} \rho_{L}(y) d y \\
& =\frac{\|G\|_{L^{\infty}}}{\varepsilon\left(L_{0} / 2\right)}
\end{aligned}
$$

which yields

$$
u_{L}(x)<\left(\frac{\|G\|_{L^{\infty}}}{\varepsilon\left(L_{0} / 2\right)}\right)^{2}
$$

which proves the assertion. 
Step 3: Uniform Coercivity of $u_{L}$ NeAR zero. Let $L_{0} \in(0,+\infty)$ be fixed. The eigenvalue property of $u_{L}$ and $\varepsilon(L)<1$ for all $L \in(0,+\infty)$ implies

$$
u_{L}^{\prime}(0)=\frac{1}{\varepsilon(L)} \int_{0}^{L}\left(G^{\prime}(-y)-G^{\prime}(y)\right) u_{L}(y) d y \geq-2 \int_{0}^{L} G^{\prime}(y) u_{L}(y) d y .
$$

Now, let $0<\delta<L$. Since $G^{\prime}$ is strictly negative on $[\delta, L]$, we have $\inf _{y \in[\delta, L]} G^{\prime}(y) \geq$ $C_{L, \delta}$ for some constant $C_{L, \delta}>0$ depending on $L$ and $\delta$. Hence

$$
u_{L}^{\prime}(0) \geq C_{L, \delta} \int_{\delta}^{L} u_{L}(y) d y .
$$

Moreover,

$$
\left|u_{L}^{\prime}(x)\right|=\frac{1}{\varepsilon(L)}\left|\int_{0}^{L}\left(G^{\prime}(x-y)-G^{\prime}(x+y)\right) u_{L}(y) d y\right| \leq \frac{2 L}{\varepsilon(L)}\left\|G^{\prime}\right\|_{L^{\infty}}\left\|u_{L}\right\|_{L^{\infty}},
$$

which means that $\left\|u_{L}^{\prime}\right\|_{L^{\infty}([0, L])}$ is uniformly bounded for $L$ belonging to a small neighborhood of $L_{0}$ because of Step 1. From now on in this step we shall extend $u_{L}$ outside $[0, L]$ with $u_{L}(x)=u_{L}(L)$ for $L \leq x \leq 2 L_{0}$. Therefore, we have defined the family of extended eigenfunctions $\mathcal{A}_{L_{0}}:=\left\{u_{L}:\left[0,2 L_{0}\right] \rightarrow[0,+\infty), \quad L \in I_{L_{0}}\right\}$, where $I_{L_{0}}$ is a (small enough) neighborhood of $L_{0}$.

Now, assume by contradiction that there exists an interval $[a, b]$ with $a<b<$ $L_{0} / 2$ such that $\liminf _{L \rightarrow L_{0}} \int_{a}^{b} u_{L}(y) d y=0$. Then, there exists a sequence $u_{L_{k}} \subset$ $\mathcal{A}_{L_{0}}$ with $L_{k} \rightarrow L_{0}$ such that $\lim _{k \rightarrow+\infty} \int_{a}^{b} u_{L_{k}}(y) d y=0$. Since $u_{L_{k}}^{\prime}$ is uniformly bounded with respect to $k$ on $\left[0,2 L_{0}\right]$ (with a possible jump discontinuity on $x=L_{k}$ ), then the family $\mathcal{A}_{L_{0}}$ is equicontinuous. Therefore, by Arzelá's theorem there exists a subsequence of $u_{L_{k}}$ converging uniformly to some $u_{\infty}$ continuous and bounded on $\left[0,2 L_{0}\right]$. We know that

$$
\varepsilon\left(L_{k}\right) u_{L_{k}}(x)= \begin{cases}\int_{0}^{L_{k}}(G(x-y)-G(x+y)) u_{L_{k}}(y) d y & \text { if } x \in\left[0, L_{k}\right], \\ \varepsilon\left(L_{k}\right) u_{L_{k}}\left(L_{k}\right) & \text { if } x \in\left[L_{k}, 2 L_{0}\right] .\end{cases}
$$

By possibly extracting a new subsequence, $\varepsilon\left(L_{k}\right)$ has a limit as $k \rightarrow+\infty$ which we call $\bar{\varepsilon}_{L_{0}}$. Therefore, the left hand side of (4.17) converges to $\bar{\varepsilon}_{L_{0}} u_{\infty}(x)$ for all $x \in\left[0, L_{0}\right]$ as $k \rightarrow+\infty$. From the uniform convergence of $u_{L_{k}}$ it is easy to see that the right hand side of (4.17) converges to

$$
\begin{cases}\int_{0}^{L_{0}}(G(x-y)-G(x+y)) u_{\infty}(y) d y & \text { if } x \in\left[0, L_{0}\right], \\ \bar{\varepsilon}_{L_{0}} u_{\infty}\left(L_{0}\right) & \text { if } x \in\left(L_{0}, 2 L_{0}\right] .\end{cases}
$$

Therefore, for $x \in\left[0, L_{0}\right]$ we have

$$
\bar{\varepsilon}_{L_{0}} u_{\infty}(x)=\int_{0}^{L_{0}}(G(x-y)-G(x+y)) u_{\infty}(y) d y .
$$

Since $u_{\infty} \geq 0$, i.e. $u_{\infty} \in K_{L_{0}}$ as defined in (4.12), by Krein-Rutman Theorem 4.10 $\bar{\varepsilon}_{L_{0}}$ must coincide with $\varepsilon\left(L_{0}\right)$ and $u_{\infty}$ is the corresponding eigenfunction of $\mathcal{H}_{L_{0}}$ in the interior of $K_{L_{0}}$. On the other hand, the uniform convergence of $u_{L_{k}}$ on $[a, b]$ implies that $\int_{a}^{b} u_{\infty}(y) d y=0$, which implies $u_{\infty} \equiv 0$ on $[a, b]$, which in turn contradicts the fact that $u_{\infty}$ lies in the interior of $K_{L_{0}}$. 
Therefore, (4.16) implies

$$
\liminf _{L \rightarrow L_{0}} u_{L}^{\prime}(0) \geq C_{L_{0}, \delta} \liminf _{L \searrow L_{0}} \int_{\delta}^{L_{0} / 2} u_{L}(y) d y=: \alpha>0,
$$

which implies that

$$
u_{L}(x)=u_{L}^{\prime}(0) x+u_{L}^{\prime \prime}(\xi) \frac{x^{2}}{2} \geq \frac{\alpha}{2} x+u_{L}^{\prime \prime}(\xi) \frac{x^{2}}{2}
$$

for some $\xi \in[0, x]$ and for $x$ in a small right-neighborhood of 0 . Since $G^{\prime \prime} \in$ $L^{\infty}\left[0, L_{0}\right]$, we have $\left\|u^{\prime \prime}\right\|_{L^{\infty}([0, L])}:=\beta<+\infty$, which implies

$$
u_{L}(x) \geq x\left(\frac{\alpha}{2}-\frac{\beta x}{2}\right)
$$

and the above right hand side is strictly positive for $x$ in a small enough neighborhood $(0, \eta]$, uniformly in $L$.

Step 4: Continuity of $\varepsilon(L)$ ON $L \in(0,+\infty)$. Steps 2 and 3 imply that we can send $\delta \searrow 0$ in (4.15). The term $\int_{0}^{L} u_{L}(x) u_{L+\delta}(x) d x$ stays away from zero uniformly for small $\delta$, whereas the term on the right hand side of (4.15) is uniformly bounded. This proves that $\lim _{L \searrow L_{0}} \varepsilon(L)=\varepsilon\left(L_{0}\right)$. By suitably changing the notation in (4.15) we also obtain

$$
(\varepsilon(L)-\varepsilon(L-\delta)) \int_{0}^{L-\delta} u_{L-\delta}(x) u_{L}(x) d x=\int_{L-\delta}^{L} d x \int_{0}^{L-\delta} d y H(x, y) u_{L-\delta}(y) u_{L}(x),
$$

which can be used to prove that $\lim _{L \nearrow L_{0}} \varepsilon(L)=\varepsilon\left(L_{0}\right)$.

Let us finally prove (ii). Assume by contradiction that

$$
\lim _{L \rightarrow+\infty} \varepsilon(L)=\varepsilon_{0}<1 .
$$

Let $\varepsilon \in\left(\varepsilon_{0}, 1\right)$. We know from Theorem 3.6 that there exists a minimizer $\rho_{\varepsilon}$ for the energy $E$ with zero center of mass. We also know that the support of $\rho_{\varepsilon}$ is compact from Lemma 4.6. From Proposition 4.3 we know that $\rho_{\varepsilon}$ is symmetric and monotonically decreasing on $x>0$. Therefore, $\rho_{\varepsilon}$ is the unique eigenfunction with unit mass provided by Proposition 4.11, and the support of $\rho_{\varepsilon}$ is $[-L, L]$ for some $L>0$. Therefore, the corresponding eigenvalue should be $\varepsilon(L)<\varepsilon_{0}$, which is a contradiction since $\varepsilon$ and $\varepsilon_{0}$ are two different eigenvalues with the same eigenfunction.

Let us prove (iii). By letting $L \searrow 0$ one has that the operator norm of $\mathcal{H}_{L}: L^{\infty}([0, L]) \rightarrow L^{\infty}([0, L])$ is arbitrarily small. Since $\varepsilon(L)$ is the spectral radius of $\mathcal{H}_{L}, \varepsilon(L)$ can be made arbitrarily small, thus contradicting the fact that $\lim _{L \searrow 0} \varepsilon(L)>0$.

We are now ready to prove the main result of this paper.

TheOrem 4.13. Let $\varepsilon<1$. Then, there exists a unique $\rho \in L^{2}$ solution to

$$
\rho \partial_{x}(\varepsilon \rho-G * \rho)=0,
$$

with unit mass and zero center of mass. Moreover, 
- $\rho$ is symmetric and monotonically decreasing on $x>0$;

- $\rho \in C^{2}(\operatorname{supp}[\rho])$;

- $\operatorname{supp}[\rho]$ is a bounded interval in $\mathbb{R}$;

- $\rho$ has a global maximum at $x=0$ and $\rho^{\prime \prime}(0)<0$;

- $\rho$ is the global minimizer of the energy $E[\rho]=\frac{\varepsilon}{2} \int \rho^{2} d x-\frac{1}{2} \int \rho G * \rho d x$.

Proof. We know from Theorem 3.6 that there exists a minimizer $\rho_{\infty}$ with unit mass and zero center of mass, which is symmetric and monotonically decreasing on $x>0$ (in view of Proposition 4.3) and compactly supported on a certain $[-L, L]$ (in view of Lemma 4.6). From the results in Proposition 4.11 and 4.12 , we know that there exists a unique steady state with such properties, because the correspondence $\varepsilon=\varepsilon(L)$ is one-to-one. So, the only possibility to violate uniqueness of steady states with unit mass and zero center of mass is to have a steady state which violates either the monotonicity property or the symmetry. Suppose first that there exists a steady state with zero center of mass $\rho$ which is not symmetric; it is not restrictive to assume that the support of $\rho$ is $\left[-L^{\prime}, L^{\prime}\right]$. Then, we know from Lemma 4.7 that it is possible to construct a symmetric steady state $\widetilde{\rho}$ with the same energy of $\rho$ and with the same support of $\rho$. Now, there are two possibilities: either $\widetilde{\rho}$ is a minimizer or not. In the former case $\rho$ is also a minimizer and this is a contradiction (a minimizer is symmetric). In the latter case, $L^{\prime}<L$ in view of Lemma 4.8 , and $\widetilde{\rho}$ is not monotonically decreasing on $x>0$ because otherwise it would be the unique minimizer provided before. Therefore, with the notation of Proposition 4.11, $-\widetilde{\rho}^{\prime}$ is an eigenfunction for $\mathcal{H}_{L^{\prime}}$ in the space $X_{L^{\prime}}$ which does not belong to the solid cone $K_{L^{\prime}}$. Therefore, the stronger version of Krein-Rutman's Theorem 4.10 and the fact that $\varepsilon(L)$ is increasing imply that $-\widetilde{\rho}^{\prime}$ is an eigenfunction outside the solid cone $K_{L^{\prime}}$, with eigenvalue strictly less than $\varepsilon\left(L^{\prime}\right)$, i.e. $\varepsilon(L)<\varepsilon\left(L^{\prime}\right)$ and therefore $L<L^{\prime}$, which contradicts $L>L^{\prime}$. The case in which $\rho$ is symmetric but not monotonic on $x>0$ can be covered by repeating the same argument above (assume $\rho=\widetilde{\rho}$ !).

Corollary 4.14 (Concavity of $\rho$ for small $\varepsilon$ ). There exists a value $\varepsilon_{0} \in$ $(0,1)$ such that, for all $\varepsilon \in\left(0, \varepsilon_{0}\right)$ the corresponding stationary solution provided in Theorem 4.13 is concave on the whole interval $[0, L]$.

Proof. We can differentiate twice with respect to $x$ in

$$
\varepsilon \rho(x)=\int_{-L}^{L} G(x-y) \rho(y) d y+C
$$

to obtain

$$
\varepsilon \rho^{\prime \prime}(x)=\int_{-L}^{L} G^{\prime \prime}(x-y) \rho(y) d y
$$

for all $x \in[-L, L]$. Therefore, $G^{\prime \prime}$ is evaluated on the interval $[-2 L, 2 L]$ in the above integral. We know from Proposition 4.12 that $L$ is a monotonically increasing function of $\varepsilon$ with $\lim _{\varepsilon \searrow 0} L(\varepsilon)=0$. Since $G^{\prime \prime}(0)<0$ and $G \in C^{2}$, there exists $L_{0}>0$ such that $G^{\prime \prime}<0$ on $\left[-2 L_{0}, 2 L_{0}\right]$. Let $\varepsilon_{0}$ be the eigenvalue in $K$ corresponding to $L=L_{0}$. Then, the eigenfunction $\rho$ is concave on its support.

REMARK 4.15 (The case meas $(\operatorname{supp}(G))<+\infty$ ). It is also interesting to consider the case with the support of $G$ being bounded, i.e. $\operatorname{supp}(G)=[-g, g]$ with 
$G$ being symmetric and monotone on $[-g, 0]$. Then most techniques of the paper remain valid, in particular when considering simply connected stationary states, whose existence can be shown along the lines of the above arguments. With the same arguments as in the proof of Lemma 4.1, one can show that the distance between two connected components of a stationary solution is at least $2 g$. This means that stationary solutions consist of a countable number of connected components, which are not influenced by any other connected component (the distance is larger than the kernel range). Thus, each connected component is a stationary solution by itself, and in particular we can prove the existence of infinitely many stationary states. The remaining step would be to characterize the behavior of each connected component as a unique energy minimizer. All arguments above apply to the case of simply connected solutions in the case of a finite range kernel $G$, except the application of the strong version of the Krein-Rutman Theorem. If we cannot guarantee that the support of the global minimizer is contained in $\left[-\frac{g}{2}, \frac{g}{2}\right]$, then there can be elements in the nonnegative cone such that the convolution with $G$ is not strictly positive. Although we strongly believe that such a smallness of the support holds at least for $\epsilon$ small, we so far have not succeeded in proving such a result.

\section{Numerical results}

In the following we show numerical simulations for the evolution equation (2.1). We discretize the equation using an explicit Euler scheme and finite difference methods. In one dimension, we partitionate the domain $\Omega=[a, b]$ using an equidistant grid with $n+1$ grid points $a=x_{0}<x_{1}<\ldots<x_{n}=b$ and step size $h=(b-a) /(n+1)$. Furthermore we use the following finite difference scheme:

$$
\frac{\rho_{i}^{j+1}-\rho_{i}^{j}}{d t}=D_{-}^{x}\left(\rho_{i}^{j} D_{+}^{x}\left(\epsilon \rho_{i}^{j}-G * \rho_{i}^{j}\right)\right)
$$

with forward and backward difference quotients

$$
D_{+}^{x} \rho_{i}=\frac{\rho_{i+1}-\rho_{i}}{h}, \quad D_{-}^{x} \rho_{i}=\frac{\rho_{i}-\rho_{i-1}}{h} .
$$

The time step size $d t$ must be chosen appropriately in order to guarantee stability. In a first example we consider an interaction potential $G(x)=$ $\frac{1}{\sigma \sqrt{2 \pi}} \exp \left(-\frac{1}{2}\left(\frac{x-\mu}{\sigma}\right)^{2}\right)$, with mean $\mu=0$ and variance $\sigma=1$, which fulfill the conditions (1)-(8), $\|G\|_{L^{1}}=1$, and models a wide range attraction.

For this kernel we present in figure 5.1 the solutions for the stationary equation (2.2), which means that we calculated the largest eigenvalues and corresponding eigenvectors of the operator $\mathcal{G}_{L}$ defined in (4.11) for different $L$. The largest eigenvalues $\epsilon=\epsilon(L)$ are presented in figure 5.1 (a). As mentioned in Proposition 4.12, $\epsilon(L)$ is strictly increasing with respect to $L$, and furthermore $\lim _{L \rightarrow \infty} \epsilon(L)=1$. The corresponding eigenfunctions with unit mass are presented in figure 5.1 (b) and (c). We proved in Corollary 4.14 the concavity of $\rho$ for small $\epsilon$. To better clarify the situation, we illustrate in figure 5.1 (b) the eigenfunctions for $L \in(0,1]$, i.e. for $\epsilon<0.05$. For a certain $\epsilon$, which depends on the concavity of the kernel $G$, the solution is not fully concave on its support, but bell shaped; compare with figure 5.1 (c).

To make this result more clear we present in figure 5.2 the stationary solutions of the evolution equation (2.1) for $\epsilon \in(0,1)$. We consider a compactly supported 
734 QUADRATIC DIFFUSION EQUATIONS WITH LONG-RANGE ATTRACTION

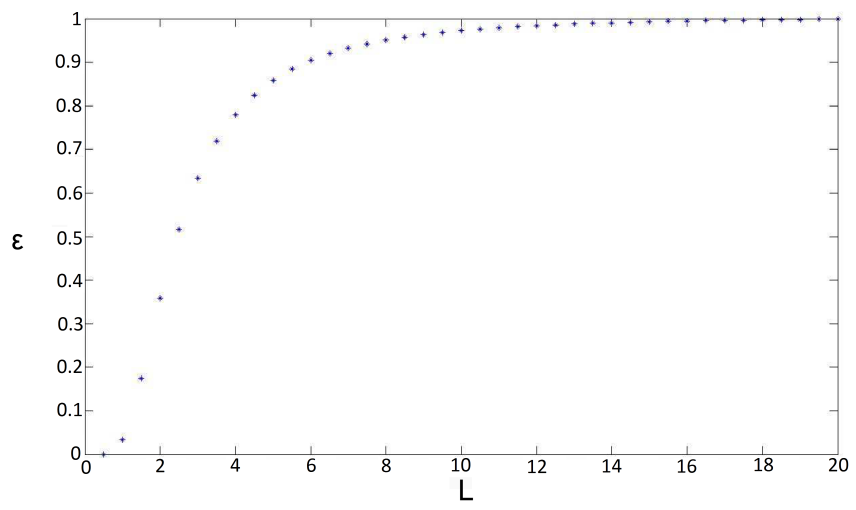

(a)

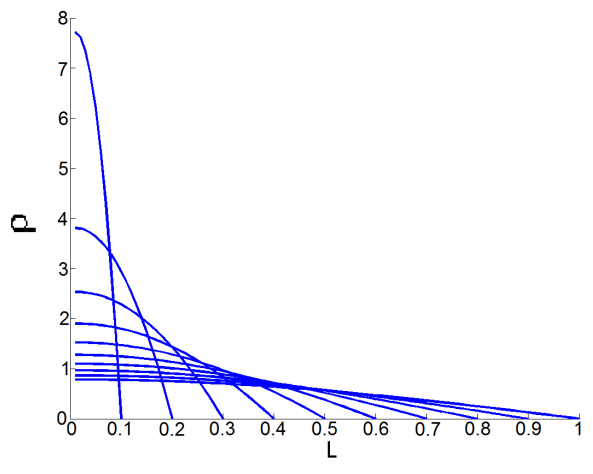

(b)

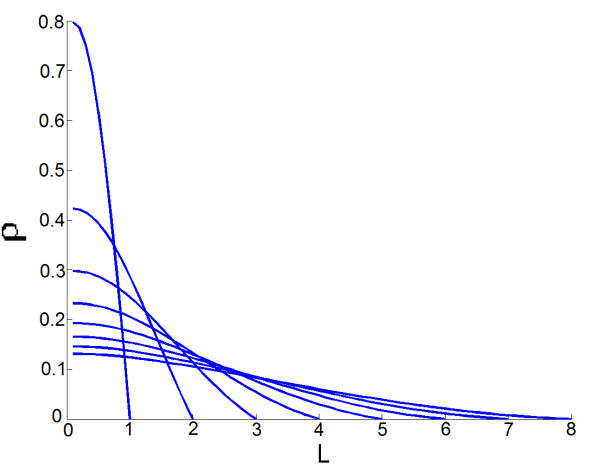

(c)

FIG. 5.1. Largest eigenvalues and corresponding eigenfunctions of the operator $\mathcal{G}_{L}$ 4.11: (a) Largest eigenvalues $\epsilon=\epsilon(L)$ of $\mathcal{G}_{L}$ on $L=(0,20]$; (b) Corresponding eigenfunctions for $\epsilon(L)$ with $L \in[0,1]$; (c) Corresponding eigenfunctions for $\epsilon(L)$ with $L \in[1,8]$.

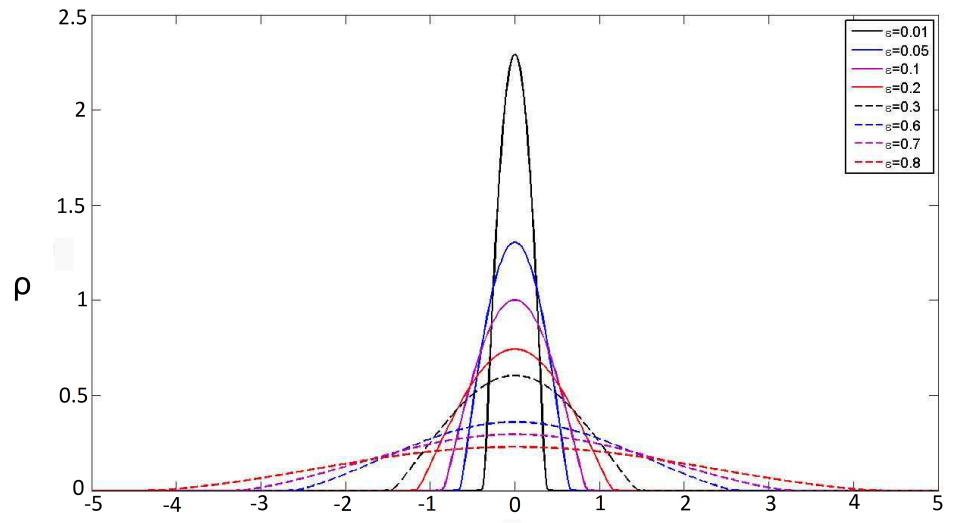

FIG. 5.2. Stationary solutions for equation (2.1) with $\epsilon \in(0,1)$. 


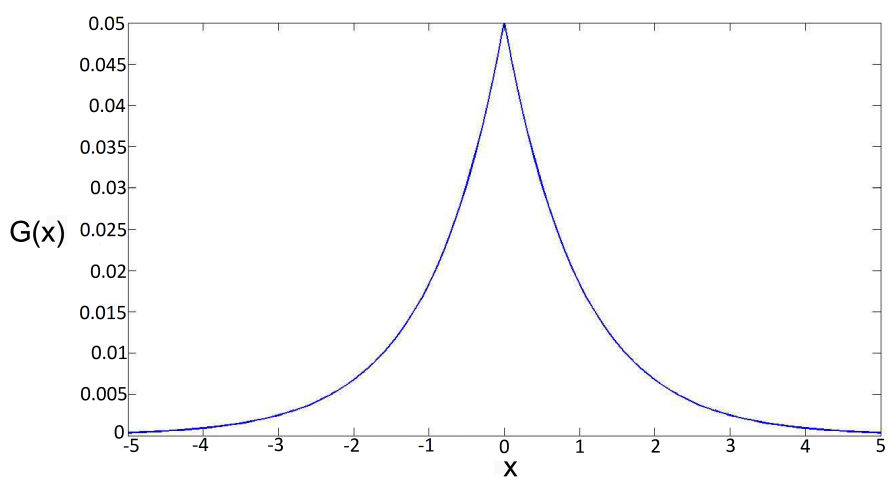

(a)

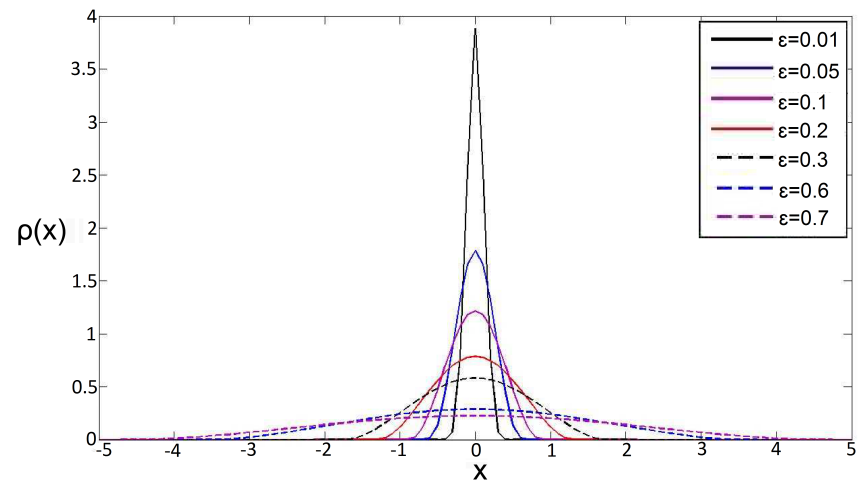

(b)

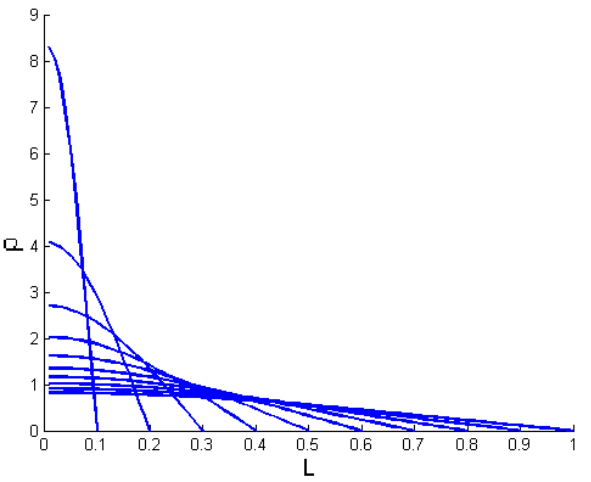

(c)

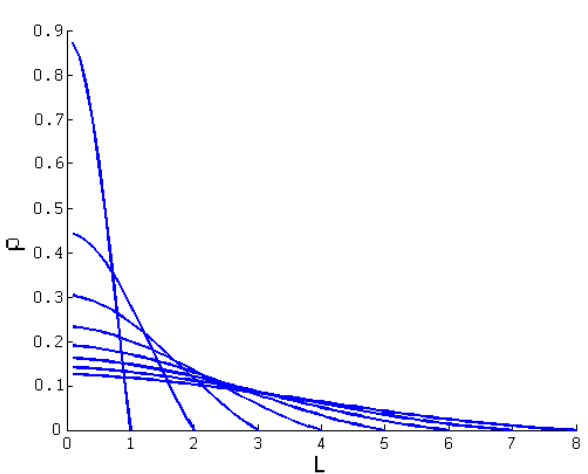

(d)

FIG. 5.3. (a) Kernel $G(x)=\frac{1}{2} \exp (-|x|)$; (b) Stationary solutions for equation (2.1) for the kernel $G$. (c),(d) eigenfunctions for $\epsilon(L)$ with $L \in[0,1]$ resp. $L \in[1,8]$. 
initial datum $\rho(x, 0)=\rho_{0}$ with unit mass $\int_{\Omega} \rho_{0}=1$. As the results are the same as in figure 5.1 (b)-(c), we recognize again that up to a certain $\epsilon$ the solutions are concave and then bell shaped. Furthermore we have mass conservation. As proven before in this paper, for $\epsilon \geq \int G=1$ we do not have steady states because the impact of the diffusive term is higher then that of the aggregation term. In this case we expect the solutions to behave like the self-similar Barenblatt-Pattle profiles. For $\epsilon=0$ we obtain an unique stationary solution (with zero center of mass), which is a Dirac- $\delta$-distribution with unit mass centered at zero.

Furthermore we computed the stationary solutions (2.1) with the kernel $G(x)=\frac{1}{2} \exp (-|x|)$ (see figure 5.3(a)) with Lipschitz singularity at the point zero. We present the results in figure 5.3(b) for different $\epsilon$. Additionally we present eigenfunctions for the corresponding operator $\mathcal{G}_{L} 4.11$ in figure 5.3(c),(d).

Acknowledgement. MDF is supported by the 'Ramon y Cajal' Subprogramme (MICINN-RYC) of the Spanish Ministry of Science and Innovation, Ref. RYC-2010-06412 and by the Ministerio de Ciencia e Innovación, Grant MTM2011-27739-C04-02. Most of the work of this paper was carried out during two visits by MDF at the Institute for Computational and Applied Mathematics of the University of Muenster. He is very grateful to MB and his group for their support and hospitality. MB and MDF thank the Isaac Newton Institute, Cambridge, for hospitality and financial support during the programme Partial Differential Equations in Kinetic Theory.

\section{REFERENCES}

[1] L. Ambrosio, N. Gigli, and G. Savaré, Gradient Flows in Metric Spaces and in the Space of Probability Measures, Lectures in Mathematics, ETH Zürich. Basel: Birkhäuser, Second Edition, 2008.

[2] D. Balagué, J.A. Carrillo, T. Laurent, and G. Raoul, Nonlocal interactions by repulsiveattractive potentials: Radial ins/stability, UAB, to appear in Physica D, 2011.

[3] J. Bedrossian, Global minimizers for free energies of subcritical aggregation equations with degenerate diffusion, Appl. Math. Letters, 24, 1927-1932, 2011.

[4] D. Benedetto, E. Caglioti, and M. Pulvirenti, A kinetic equation for granular media, RAIRO Modél. Math. Anal. Numér., 31, 615-641, 1997.

[5] A. Bertozzi, J. Carrillo, and T. Laurent, Blowup in multidimensional aggregation equations with mildly singular interaction kernels, Nonlin., 22, 683-710, 2009.

[6] A. Bertozzi and T. Laurent, Finite-time blow-up of solutions of an aggregation equation in $\mathbb{R}^{n}$, Commun. Math. Phys., 274, 717-735, 2007.

[7] A.L. Bertozzi and J. Brandman, Finite-time blow-up of $L^{\infty}$-weak solutions of an aggregation equation, Commun. Math. Sci., 8(1), 45-65, 2010.

[8] A.L. Bertozzi, T. Laurent, and J. Rosado, $L^{p}$ theory for the multidimensional aggregation equation, Commun. Pure Appl. Math., 64(1), 45-83, 2011.

[9] A.L. Bertozzi and D. Slepčev, Existence and uniqueness of solutions to an aggregation equation with degenerate diffusion, Commun. Pure Appl. Anal., 9, 1617-1637, 2010.

[10] A. Blanchet, J. Dolbeault, and B. Perthame, Two-dimensional keller-segel model: Optimal critical mass and qualitative properties of the solutions, Electron. J. Differ. Eqs., (44), 1-32, (electronic), 2006.

[11] M. Bodnar and J. Velazquez, An integro-differential equation arising as a limit of individual cell-based models, J. Diff. Equ., 222(2), 341-380, 2006.

[12] S. Boi, V. Capasso, and D. Morale, Modeling the aggregative behavior of ants of the species Polyergus rufescens, Nonlin. Anal., Real World Appl., 1(1), 163-176, 2000.

[13] M. Burger, V. Capasso, and D. Morale, On an aggregation model with long and short range interactions, Nonlin. Anal., Real World Appl., 8(3), 939-958, 2007.

[14] M. Burger and M. Di Francesco, Large time behavior of nonlocal aggregation models with nonlinear diffusion, Netw. Heterog. Media, 3(4), 749-785, 2008.

[15] M. Burger, M. Di Francesco, and Y. Dolak-Struß, The Keller-Segel model for chemotaxis 
with prevention of overcrowding: Linear vs. nonlinear diffusion, SIAM J. Math. Anal., 38, 1288-1315, 2006.

[16] V. Calvez and J. Carrillo, Volume effects in the Keller-Segel model: Energy estimates preventing blow-up, J. Math. Pures Appl., 86, 155-175, 2006.

[17] J. Carrillo, M. Di Francesco, A. Figalli, T. Laurent, and D. Slepčev, Global-in-time weak measure solutions, and finite-time aggregation for nonlocal interaction equations, Duke Math. J., 156, 229-271, 2011.

[18] J. Carrillo, M. Di Francesco, A. Figalli, T. Laurent, and D. Slepčev, Confinement in nonlocal interaction equations, Nonlin. Anal., 75, 550-558, 2012.

[19] J. Carrillo, R. McCann, and C. Villani, Kinetic equilibration rates for granular media and related equations: Entropy dissipation and mass transportation estimates, Rev. Mat. Iberoam., 19(3), 971-1018, 2003.

[20] J. Carrillo, R. McCann, and C. Villani, Contractions in the 2-Wasserstein length space and thermalization of granular media, Arch. Ration. Mech. Anal., 179(2), 217-263, 2006.

[21] J.A. Carrillo and G. Toscani, Asymptotic $L^{1}$-decay of solutions of the porous medium equation to self-similarity, Indiana Univ. Math. J., 49, 113-141, 2000.

[22] J. Cates, P.T. Fletcher, M. Styner, M. Shenton, and R. Whitaker, Shape modeling and analysis with entropy-based particle systems, Information Processing in Medical Imaging IPMI, LNCS, 4584, 333-345, 2007.

[23] L. Chayes, I. Kim, and Y. Yao, An aggregation equation with degenerate diffusion: Qualitative property of solutions, preprint.

[24] Y.L. Chuang, M.R. D’Orsogna, D. Marthaler, A.L. Bertozzi, and L.S. Chayes, State transitions and the continuum limit for a $2 D$ interacting, self-propelled particle system, Physica D, 232(1), 33-47, 2007.

[25] L. Corrias, B. Perthame, and H. Zaag, Global solutions of some chemotaxis and angiogenesis systems in high space dimensions, Milan J. Math., 72, 1-28, 2004.

[26] H. Dirks, Medical Image Segmentation Including Particle Methods, Diploma Thesis, WWU Münster, 2011.

[27] M.R. D’Orsogna, Y.L. Chuang, A.L. Bertozzi, and L.S. Chayes, Self-propelled particles with soft-core interactions: Patterns, stability and collapse, Phys. Rev. Lett., 96, 2006.

[28] Y. Du, Order Structure and Topological Methods in Nonlinear Partial Differential Equations, Vol. 1: Maximum Principles and Applications, Series in Partial Differential Equations and Applications: Volume 2, Hackensack, New Jersey, World Scientific, 2006.

[29] K. Fellner and G. Raoul, Stability of stationary states of non-local equations with singular interaction potentials, Math. Comput. Modelling, 53(7-8), 1436-1450, 2011.

[30] K. Fellner and G. Raoul, Stable stationary states of non-local interaction equations, Math. Models Methods Appl. Sci., 20(12), 2267, 2010.

[31] R.C. Fetecau, Y. Huang, and T. Kolokolnikov, Swarm dynamics and equilibria for a nonlocal aggregation model, Nonlin., 24, 2681-2716, 2011.

[32] M. Fornasier, J. Haškovec, and G. Steidl, Consistency of variational continuous-domain quantization via kinetic theory, Appl. Anal., to appear, 2012.

[33] F. Golse, The mean-field limit for the dynamics of large particle systems, Proceedings of the Conference on Partial Differential Equations, Forges-les-Eaux, France, June 2-6, 2003.

[34] M.Z. Guo, G.C. Papanicolaou, and S.R.S. Varadhan, Nonlinear diffusion limit for a system with nearest neighbor interactions, Commun. Math. Phys., 118, 31-59, 1988.

[35] M.A. Herrero and J.J.L. Velázquez, Singularity patterns in a chemotaxis model, Math. Ann., 306(3), 583-623, 1996.

[36] T. Hillen and K. Painter, Global existence for a parabolic chemotaxis model with prevention of overcrowding, Adv. Appl. Math., 26(4), 280-301, 2001.

[37] Y. Huang and A.L. Bertozzi, Self-similar blow-up solutions to an aggregation equation in $\mathbb{R}^{n}$, SIAM J. Appl. Math., 70(7), 2582-2603, 2010.

[38] W. Jäger and S. Luckhaus, On explosions of solutions to a system of partial differential equations modelling chemotaxis, Trans. Amer. Math. Soc., 329(2), 819-824, 1992.

[39] K. Kang, B. Perthame, A. Stevens, and J. Velázquez, An integro-differential equation model for alignment and orientational aggregation, J. Differ. Eqs., 246(4), 1387-1421, 2009.

[40] E.F. Keller and L.A. Segel, Initiation of slide mold aggregation viewed as an instability, J. Theor. Biol., 26, 399-415, 1970. 
[41] I. Kim and Y. Yao, The Patlak-Keller-Segel model and its variations: Properties of solutions via maximum principle, SIAM J. Math. Anal., 44(2), 568-602, 2012.

[42] R. Kowalczyk, Preventing blow-up in a chemotaxis model, J. Math. Anal. Appl., 305(2), $566-588,2005$.

[43] P. Laurençot and D. Wrzosek, A chemotaxis model with threshold density and degenerate diffusion, in Nonlinear Elliptic and Parabolic Problems, Progr. Nonlin. Diff. Equ. Appl., Birkhäuser, Basel, 64, 273-290, 2005.

[44] T. Laurent, Local and global existence for an aggregation equation, Commun. Part. Differ. Eqs., 32(12), 1941-1964, 2007.

[45] D. Li and X. Zhang, On a nonlocal aggregation model with nonlinear diffusion, Discrete Contin. Dyn. Syst., 27(1), 301-323, 2010.

[46] H. Li and G. Toscani, Long-time asymptotics of kinetic models of granular flows, Arch. Ration. Mech. Anal., 172(3), 407-428, 2004.

[47] E.H. Lieb, Sharp constants in the Hardy-Littlewood-Sobolev and related inequalities, Ann. Math., 118, 349-374, 1983.

[48] E.H. Lieb and M. Loss, Analysis, Graduate Studies in Mathematics, American Mathematical Society, Providence, RI, 14, 1997.

[49] P.L. Lions, The concentration-compactness principle in calculus of variations, in The Locally Compact Case, Ann. Inst. Henri Poincaré, Anal. Nonlin., 1, 109-145, 1984.

[50] R.J. McCann, A convexity principle for interacting gases, Adv. Math., 128(1), 153-179, 1997.

[51] P.A. Milewski and X. Yang, A simple model for biological aggregation with asymmetric sensing, Commun. Math. Sci., 6, 397-416, 2008.

[52] A. Mogilner and L. Edelstein-Keshet, A non-local model for a swarm, J. Math. Biol., 38(6), 534-570, 1999.

[53] D. Morale, V. Capasso, and K. Oelschlaeger, An interacting particle system modelling aggregation behavior: From individuals to populations, J. Math. Biol., 50(1), 49-66, 2005.

[54] K. Ölschläger, Large systems of interacting particles and porous medium equation, J. Diff. Eqs., 88, 294-346, 1990.

[55] F. Otto, The geometry of dissipative evolution equations: The porous medium equation, Commun. Part. Differ. Eqs., 26(1-2), 101-174, 2001.

[56] K. Painter and T. Hillen, Volume-filling and quorum sensing in models for chemosensitive movement, Canadian Applied Mathematics Quaterly, 10(4), 280-301, 2003.

[57] C. Patlak, Random walk with persistence and external bias, Bull. Math. Biophys., 15, 311-338, 1953.

[58] M. Porfiri, D. Stilwell, and E. Bollt, Decline of minorities in stubborn societies, Eur. Phys. J. B: Condensated Matter and Complex Systems, 57, 481-486, 2007.

[59] I. Primi, A. Stevens, and J. Velázquez, Mass-selection in alignment models with nondeterministic effects, Commun. Part. Differ. Eqs., 34(5), 419-456, 2009.

[60] K. Sznajd-Weron and J. Sznajd, Opinion evolution in closed community, Int. J. Mod. Phys. C, 11, 1157-1165, 2000.

[61] C.M. Topaz and A.L. Bertozzi, Swarming patterns in a two-dimensional kinematic model for biological groups, SIAM J. Appl. Math., 65(1), 152-174, 2004.

[62] C.M. Topaz, A.L. Bertozzi, and M. Lewis, A nonlocal continuum model for biological aggregation, Bull. Math. Bio., 68(7), 1601-1623, 2006.

[63] S.R.S. Varadhan, Scaling limits for interacting diffusions, Commun. Math. Phys., 135, 313-353, 1991.

[64] J.L. Vázquez, The Porous Medium Equation, Oxford Mathematical Monographs. The Clarendon Press Oxford University Press, Oxford, 2007.

[65] C. Villani, Topics in Optimal Transportation, Graduate Studies in Mathematics, American Mathematical Society, Providence, RI, 58, 2003.

[66] C. Villani, Optimal Transport. Old and New, Grundlehren der Mathematischen Wissenschaften, Berlin: Springer, 338, 2008. 